\title{
Fast Quaternion Product Units for Learning Disentangled Representations in $\mathrm{SO}(3)$
}

This paper was downloaded from TechRxiv (https://www.techrxiv.org).

LICENSE

CC BY 4.0

SUBMISSION DATE / POSTED DATE

04-01-2022 / 07-01-2022

CITATION

Qin, Shaofei; Zhang, Xuan; Xu, Hongteng; Xu, Yi (2022): Fast Quaternion Product Units for Learning Disentangled Representations in SO(3). TechRxiv. Preprint. https://doi.org/10.36227/techrxiv.17791574.v1

DOI

10.36227/techrxiv.17791574.v1 


\title{
Fast Quaternion Product Units for Learning Disentangled Representations in $\mathbb{S O}(3)$
}

\author{
Shaofei Qin, Xuan Zhang, Hongteng Xu, Member, IEEE, and Yi Xu, Member, IEEE
}

\begin{abstract}
Real-world 3D structured data like point clouds and skeletons often can be represented as data in a 3D rotation group (denoted as $\mathbb{S O}(3)$ ). However, most existing neural networks are tailored for the data in the Euclidean space, which makes the 3D rotation data not closed under their algebraic operations and leads to sub-optimal performance in 3D-related learning tasks. To resolve the issues caused by the above mismatching between data and model, we propose a novel non-real neuron model called quaternion product unit (QPU) to represent data on 3D rotation groups. The proposed QPU leverages quaternion algebra and the law of the 3D rotation group, representing $3 \mathrm{D}$ rotation data as quaternions and merging them via a weighted chain of Hamilton products. We demonstrate that the QPU mathematically maintains the $S O(3)$ structure of the 3D rotation data during the inference process and disentangles the 3D representations into "rotation-invariant" features and "rotation-equivariant" features, respectively. Moreover, we design a fast QPU to accelerate the computation of QPU. The fast QPU applies a tree-structured data indexing process, and accordingly, leverages the power of parallel computing, which reduces the computational complexity of QPU in a single thread from $\mathcal{O}(N)$ to $\mathcal{O}(\log N)$. Taking the fast QPU as a basic module, we develop a series of quaternion neural networks (QNNs), including quaternion multi-layer perceptron (QMLP), quaternion message passing (QMP), and so on. In addition, we make the QNNs compatible with conventional real-valued neural networks and applicable for both skeletons and point clouds. Experiments on synthetic and real-world 3D tasks show that the QNNs based on our fast QPUs are superior to state-of-the-art real-valued models, especially in the scenarios requiring the robustness to random rotations.
\end{abstract}

Index Terms-Quaternion product units, quaternion neural networks, disentangled representation, rotation invariance and equivariance, $3 \mathrm{D}$ rotation modeling.

\section{INTRODUCTION}

$\mathrm{R}$ EPRESENTING 3D structured data like point clouds and skeletons is essential for many real-world applications, such as autonomous driving [1], [2], robotics [3], and gaming [4], [5]. In practice, we often model these $3 \mathrm{D}$ structured data as the collection of points in a $3 \mathrm{D}$ rotation group $\mathbb{S O}(3)$, such as representing a skeleton by the rotations between adjacent joints and representing a point cloud by the rotations from each center point to its neighbors. On the one hand, the rotations within each 3D structured data are informative and distinguishable, e.g., for the skeletons corresponding to different actions, their joints are often associated with various rotations. On the other hand, however, the uncertainty hidden in the data is also caused by the randomness of geometric transformations, especially the global rotation. For example, in action recognition, the human skeletons with different orientations may represent the same action [6], [7]; in autonomous

- M. Shaofei Qin is with the MoE Key Lab of Artificial Intelligence, AI Institute, Shanghai Jiao Tong University, Shanghai, China.

E-mail: sufer_qin@sjtu.edu.cn.

- Xuan Zhang is with the Department of Computer Science \& Engineering, Texas A\&M University, College Station, TX 77843, USA. Part of the work was done while he was with SJTU.

E-mail: xuan.zhang@tamu.edu.

- Hongteng Xu is the co-corresponding author of this paper. He is with the Gaoling School of Artificial Intelligence, Renmin University of China and Beijing Key Laboratory of Big Data Management and Analysis Methods, Beijing, China.

E-mail: hongtengxu@ruc.edu.cn.

- $\quad Y i X u$ is the co-corresponding author of this paper. She is with the MoE Key Lab of Artificial Intelligence, AI Institute, Shanghai Jiao Tong University, Shanghai, China.

E-mail:xuyi@sjtu.edu.cn.

Manuscript received XX, 2021. driving, the point clouds with different directions may capture the same vehicle [2]. Facing the 3D structured data with such rotation discrepancies, we often require the corresponding representation methods to be equivariant to informative rotations while invariant to redundant rotations.

Currently, many deep learning-based representation methods have made efforts to enhance their generalization power and robustness to rotations [8], [9], [10], [11], [12]. Unfortunately, most existing methods suffer from the following two drawbacks. Firstly, their models focus on the data in the Euclidean space, rather than in $\mathbb{S O}(3)$. As a result, the 3D rotations are not closed under the algebraic operations used in their models (i.e, additions and multiplications). ${ }^{1}$ The mismatching between data and model makes these methods challenging to analyze the influence of rotations on their outputs quantitatively. Although this problem can be mitigated by augmenting training data with additional rotations [13], this solution gives us no theoretical guarantee. Secondly, most existing methods pursue either the rotation-invariance or the rotation-equivariance when learning representations in $\mathbb{S O}(3)$. Few of them consider developing a unified representation model to disentangle these two properties explicitly. Therefore, these methods are specialized for different application scenarios and generally lead to sub-optimal results when the tasks require a trade-off between the rotation-invariance and the rotation-equivariance.

Motivated by the above challenges, we propose a novel nonreal neuron model called quaternion product unit (QPU), which

1. For example, adding two $3 \mathrm{D}$ rotation matrices together or multiplying a scalar with a $3 \mathrm{D}$ rotation matrix will not result in a valid rotation matrix. 


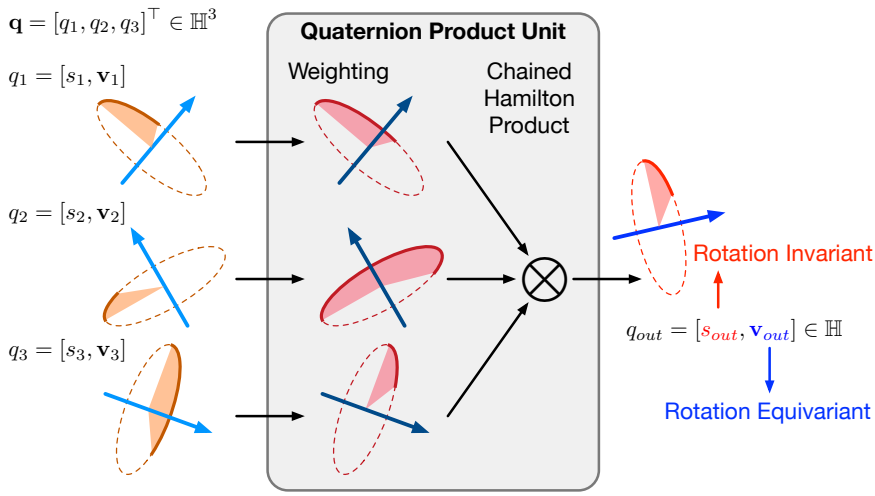

Fig. 1: An illustration of our quaternion product unit. Each rotation in $\mathbb{S O}(3)$ can be represented via a unit quaternion in a hypercomplex space $\mathbb{H}$, whose real part and imaginary part indicates the rotation angle and the direction of the rotation axis, respectively. The QPU merges input rotations via a weighted chain of Hamilton products and derives a rotation as its output, whose real part and imaginary part yield to the rotation-invariance and the rotation-equivariance, respectively.

leverages quaternion and its operations in an efficient and interpretable way to represent the data in $\mathbb{S O}(3)$. As illustrated in Figure 1, for each 3D rotation, we represent it as a unit quaternion, whose imaginary part indicates the direction of its rotation axis, and the real part corresponds to the cosine of its rotation angle, respectively. Taking $N$ quaternions as inputs, the QPU first applies quaternion power operation to each input to scale their rotation angles. Then, it applies a chain of Hamilton products to merge the weighted quaternion features and output a rotation accordingly. The parameters of the QPU consist of the scaling coefficients and the bias introduced to the rotation angles. All the operations of QPU, including weighting and chained product, are closed to $\mathbb{S O}(3)$, so it can maintain the quaternion structure and the meaning of rotation during the inference process. It should be noted that the proposed QPU achieves disentangled representations in $\mathbb{S O}(3)$. In particular, we demonstrate that the quaternion derived by QPU is disentangled naturally into a rotation-invariant real part and a rotation-equivariant imaginary part, which can support different downstream learning tasks.

We make great efforts to make the above QPU applicable in practice. With the help of a tree-structured data indexing process and a parallel computing algorithm, we develop a fast QPU in CUDA, reducing the computational complexity of QPU in a single thread from $\mathcal{O}(N)$ to $\mathcal{O}(\log N)$. Based on the fast QPU, we build various quaternion neural networks (QNNs), e.g., quaternion multi-layer perceptron (QMLP) and quaternion message passing (QMP), for learning representations in $\mathbb{S O}(3)$. Furthermore, to make the QNNs compatible with existing data formats and realvalued models, we $i$ ) propose two preprocessing methods converting skeletons and point clouds to 3D rotations, respectively, and ii) design an angle-axis mapping layer to connect the QNNs with conventional neural networks.

In summary, the core contributions of our work are as follows:

1) A novel quaternion architecture with an efficient implementation. Our QPU is a new member of quaternion-based neural networks, which makes the first attempt to represent the data on the $3 \mathrm{D}$ rotation group from the viewpoint of quaternion algebra. We further develop an efficient algorithm to accelerate its computation, which reduces its complexity significantly and makes it applicable in practice.

2) A unified framework achieving disentangled representation. The proposed QPU (and its fast version) provides an algorithmic framework that explicitly disentangles the representation of arbitrary $3 \mathrm{D}$ rotations into a rotation-invariant part and a rotation-equivariant part. As a result, the QPU is applicable to various application scenarios.

3) Highly-compatible architectures for different neural networks and data formats. The proposed QPU-based QNNs are highly compatible with existing real-valued neural networks and applicable to various kinds of 3D data.

Experiments on both synthetic and real-world data show that the proposed QPU-based models can be trained efficiently with standard back-propagation and outperform conventional real-valued models in many applications requiring rotation robustness.

The remainder of this paper is organized as follows: Section 2 introduces some related works and its background knowledge. Section 3 introduces the proposed QPU model and its properties. Section 4 includes the detailed implementation of our fast QPU, especially its acceleration strategies. Section 5 introduces the quaternion neural networks based on the fast QPU module and discusses their compatibility. Section 6 provides the experimental results on both synthetic and real-world 3D datasets. Finally, section 7 concludes the paper and discusses some future works.

\section{Related Work}

\subsection{Typical 3D applications and learning methods}

As a typical 3D application, skeleton-based action recognition has attracted lots of attention in recent years, and many learning-based methods have been proposed to solve this problem [14], [15]. The early methods often apply "bottom-up" strategies to represent skeletons. For example, the P-LSTM in [16] represents different parts of a skeleton (e.g., torso, arms, and legs) independently and merges the representations via the long short-term memory (LSTM) module. The ST-LSTM in [17] considers the order of the joints in a skeleton and regularizes the spatio-temporal continuity of the joints during learning. Recently, more methods apply "topdown" strategies to obtain global representations of skeletons directly. The ST-GCN in [18] treats the skeleton graph as a whole and applies a graph convolutional network (GCN) to extract a global feature. Following this idea, the DGNN in [19] defines the skeleton as a directed graph spreading from the chest to the limbs and applies a directional message passing process. Besides aggregating the spatial information within each skeleton, the AGCLSTM in [20] further considers the aggregation of temporal information with the help of the LSTM model. Similarly, by treating skeleton sequences as time-directed dynamic graphs, the DDGCN in [21] further emphasizes the temporal information and considers the effective features of non-adjacent nodes, while the CTR-GCN in [22] introduces a spatiotemporal inception structure to capture multi-scale information.

Besides the methods focusing on 3D skeletons, many neural networks are designed for 3D point clouds. The PointNet [13] uses multi-layer perceptrons (MLP) to extract features of points and aggregate them by pooling layers. The PointNet++ in [23] improves the PointNet by defining the neighborhood of each point before applying the PointNet. Following the above strategy, the PointWeb in [24] analyzes the relationship of all point pairs in the neighborhood and models the contextual information of the central 
point by an adaptive feature adjustment (AFA) function. The PointConv in [25] defines a continuous convolution kernel on the relative coordinate system of points, which achieves a generalized pointwise convolution neural network $(\mathrm{CNN})$ to represent point clouds. The RS-Conv in [26] improves the PointConv by introducing a parameter-free aggregation function into the convolution kernel, which avoids redundant computations and simplifies the convolution operation greatly. The PAConv in [27] further replaces the MLP structure in PointConv with a new dynamic generative model, which can better handle the irregularity of point cloud data.

\subsection{Representations invariant/equivariant to rotations}

Essentially, the methods above aim at achieving powerful representations for 3D structured data, which are equivariant to the structural change within each data while invariant to the random rotations imposed on different data. A straightforward strategy for improving the rotation-invariance of the model is to randomly rotate the training data during the training process as a data augmentation method, thereby strengthening the network's attention to the rotation-invariant information. In addition, we can also insert a preprocessing network before the model [13], [28], which can align point clouds with different orientations. However, these data-driven methods give us no theoretical guarantee on rotationinvariance, whose performance is often unstable in practice.

Representing 3D structured data in the 3D rotation group or the Lie group provides us with a more reliable solution to pursue rotation-invariant representations. For 3D skeletons, the work in [6], [29] represents human skeletons as 3D rotations in the Lie group and achieves encouraging performance on action recognition. The LieNet in [14] utilizes similar data representation in a deep learning framework on a 3D rotation manifold, where input rotation matrices were transformed progressively by being multiplied with learnable rotation matrices. For 3D point clouds, the spherical CNNs [8], [9] project the points onto a sphere and perform convolutions in the frequency domain using spherical harmonics, while ClusterNet [11] transforms point clouds into a set of $3 \mathrm{D}$ rotations. Both of them achieve rotation-invariant features for point clouds.

Additionally, because the structural change within each 3D structured data can often be represented as the change of the rotations among its 3D points, many methods have been proposed to achieve distinguishable representations that are equivariant to the local rotations imposed on the data. For example, the 3D Steerable CNNs [10] and the TensorField Networks [12] incorporate group representation theory in 3D deep learning and learn rotationequivariant features by computing equivariant kernel basis on fields. The quaternion-based neural networks in [30], [31] also achieve rotation-equivariant representations for 3D point clouds.

To our surprise, although both the rotation-invariance and the rotation-equivariance are significant for $3 \mathrm{D}$ data representation, they are seldom considered jointly. Most existing methods treat them as two conflicting properties, which can only achieve either the rotation-invariance or the rotation-equivariance. Therefore, a natural question is whether it is possible to achieve these two important properties in a unified framework simultaneously. To our knowledge, our QPU makes the first attempt to provide a positive answer to this question with the help of quaternion algebra.

\subsection{Quaternion-based models and learning algorithms}

Quaternion is widely used in computer graphics and control theory to represent 3D rotation, which only requires four parameters to describe a rotation matrix. Recently, many efforts have been made to introduce quaternion-based models to the applications of computer vision and machine learning. Quaternion wavelet transforms (QWT) [32], [33], [34], quaternion sparse coding, and quaternion dictionary learning methods [35], [36] have been successfully applied for various image processing tasks, e.g., image denoising, super-resolution, and watermarking. The quaternion lifting schema [37] applies the SLERP [38] to extract hierarchical information in rotation data to perform gait recognition.

More recently, quaternion neural networks (QNNs) [39], [40] have produced significant advances. In [41], a QNN for color image representation is developed, which replaces multiplications in standard networks with Hamilton products and better preserves the interrelationship between different color channels. Similarly, the quaternion convolution neural network (QCNN) in [42] represents the rotations in color space with quaternions and achieves a color-sensitive image representation method. In [43], [44], [45], quaternion-based recurrent neural networks reduce the complexity of sequential models for efficient natural language processing and speech recognition. Besides the above typical examples, quaternion-based machine learning methods have been applied to other applications, such as knowledge graph modeling [46] and recommendation systems [47].

For 3D point clouds, the QNN in [30] and the quaternion capsule network in [31] are two typical rotation-equivariant representation models. For 3D skeletons, the QuaterNet [5] performs human skeleton action prediction by predicting the relative rotation of each joint in the next step with unit quaternion representation. In our previous work [48], we develop a preliminary version of the proposed quaternion product unit (QPU), which achieves encouraging performance on learning rotation-invariant skeleton representation models. In this paper, we will further study the QPU in-depth, improving its implementations and making it more applicable to various data and tasks.

\section{Proposed Quaternion Product Units}

As aforementioned, many 3D structured data can be represented as a set of 3D rotations. Take 3D skeleton data as an example. A 3D skeleton is a $3 \mathrm{D}$ point cloud associated with a graph structure. We can represent a skeleton by a set of $3 \mathrm{D}$ rotations, using relative rotations between edges (i.e., joint rotations). The 3D rotation from a vector $\boldsymbol{v}_{1} \in \mathbb{R}^{3}$ to another vector $\boldsymbol{v}_{2} \in \mathbb{R}^{3}$ could be described as a rotation around an axis $\boldsymbol{u}$ with a angle $\theta$ :

$$
\begin{aligned}
& \text { Rotation Axis: } \boldsymbol{u}=\frac{\boldsymbol{v}_{1} \times \boldsymbol{v}_{2}}{\left\|\boldsymbol{v}_{1} \times \boldsymbol{v}_{2}\right\|_{2}}, \\
& \text { Rotation Angle: } \theta=\arccos \left(\frac{\left\langle\boldsymbol{v}_{1}, \boldsymbol{v}_{2}\right\rangle}{\left\|\boldsymbol{v}_{1}\right\|_{2}\left\|\boldsymbol{v}_{2}\right\|_{2}}\right) .
\end{aligned}
$$

Here, $\boldsymbol{v}_{1} \times \boldsymbol{v}_{2}$ stands for cross-product and $\left\langle\boldsymbol{v}_{1}, \boldsymbol{v}_{2}\right\rangle$ is for innerproduct. The $3 \mathrm{D}$ rotations can be treated as points in the $3 \mathrm{D}$ rotation group $\mathbb{S O}(3)$. Mathematically, a group represents an algebraic structure consisting of a set and a dyadic operation that satisfies the closure and associative law and has the identity and inverse elements. In the following content, we will design a quaternion product unit to achieve the fusion of 3D rotations with the help of quaternion algebra.

\subsection{Quaternion and 3D rotation}

As shown in [49], [50], 3D rotations can be represented as a quaternion, and accordingly, the dyadic operation of $\mathbb{S O}(3)$ corresponds to the Hamilton product between two quaternions, denoted 
as $\otimes$. In particular, the quaternion is a type of hypercomplex number with 1D real part $s$ and 3D imaginary part $(x, y, z)$. We denote $\mathbb{H}$ as the space of quaternion and $q=s+\mathbf{i} x+\mathbf{j} y+\mathbf{k} z \in \mathbb{H}$ as an arbitrary quaternion. Here, the imaginary parts satisfy $\mathbf{i}^{2}=\mathbf{j}^{2}=$ $\mathbf{k}^{2}=\mathbf{i j} \mathbf{k}=-1, \mathbf{i j}=-\mathbf{j i}=\mathbf{k}, \mathbf{k} \mathbf{i}=-\mathbf{i k}=\mathbf{j}, \mathbf{j} \mathbf{k}=-\mathbf{k} \mathbf{j}=\mathbf{i}$. For convenience, we ignore the imaginary symbols and represent a quaternion as the combination of a scalar and a column vector as $q=\left[s, \boldsymbol{v}^{T}\right]^{T}=[s, x, y, z]^{T}$. The Hamilton product between two quaternions $q_{1}=\left[s_{1}, \boldsymbol{v}_{1}^{T}\right]^{T}$ and $q_{2}=\left[s_{2}, \boldsymbol{v}_{2}^{T}\right]^{T}$ is defined as (2):

$$
\begin{aligned}
q_{1} \otimes q_{2} & =\left[s_{1} s_{2}-\left\langle\boldsymbol{v}_{1}, \boldsymbol{v}_{2}\right\rangle, \boldsymbol{v}_{1} \times \boldsymbol{v}_{2}+s_{1} \boldsymbol{v}_{2}+s_{2} \boldsymbol{v}_{1}\right] \\
& =M_{L}\left(q_{1}\right) q_{2}=M_{R}\left(q_{2}\right) q_{1},
\end{aligned}
$$

where $M_{L}(q)$ and $M_{R}(q)$ represent two matrices constructed by a quaternion $q$ :

$$
M_{L}(q)=\left[\begin{array}{cccc}
s & -x & -y & -z \\
x & s & -z & y \\
y & z & s & -x \\
z & -y & x & s
\end{array}\right], \quad M_{R}(q)=\left[\begin{array}{cccc}
s & -x & -y & -z \\
x & s & z & -y \\
y & -z & s & x \\
z & y & -x & s
\end{array}\right] .
$$

Note that the Hamilton product is non-commutative - multiplying a quaternion on the left or the right gives different matrices. Additionally, to avoid misunderstandings, the quaternion mentioned in this article all refer to unit quaternions, which means $\|q\|=s^{2}+x^{2}+y^{2}+z^{2}=1$.

As shown in (1), any rotation in the $3 \mathrm{D}$ space can be represented as rotating an angle $\theta$ around a rotation axis $\boldsymbol{u}$. Accordingly, the quaternion corresponding to a rotation can be defined as

$$
q=\left[s, \boldsymbol{v}^{T}\right]^{T}=\left[\cos \left(\frac{\theta}{2}\right), \sin \left(\frac{\theta}{2}\right) \boldsymbol{u}^{T}\right]^{T},\|\boldsymbol{u}\|_{2}=1,
$$

and applying the rotation to a vector $\boldsymbol{v}_{1}$ can be achieved by the Hamilton products involving the corresponding quaternion:

$$
\left[0, \boldsymbol{v}_{2}^{T}\right]^{T}=q \otimes\left[0, \boldsymbol{v}_{1}^{T}\right]^{T} \otimes q^{*},
$$

where $\boldsymbol{v}_{2}$ is the result of the rotation, and $q^{*}=[s,-\boldsymbol{v}]$ stands for the conjugation of $q$.

As a natural extension of (5), the combination of rotations can also be represented by the chained Hamilton products of quaternions. Specifically, if we apply two rotations (denoted as two quaternions $q_{1}$ and $q_{2}$ ) to the vector $\boldsymbol{v}$ in turn, we have

$$
\begin{aligned}
& q_{2} \otimes\left(q_{1} \otimes\left[0, \boldsymbol{v}^{T}\right]^{T} \otimes q_{1}^{*}\right) \otimes q_{2}^{*} \\
= & \left(q_{2} \otimes q_{1}\right) \otimes\left[0, \boldsymbol{v}^{T}\right]^{T} \otimes\left(q_{1}^{*} \otimes q_{2}^{*}\right) \\
= & \left(q_{2} \otimes q_{1}\right) \otimes\left[0, \boldsymbol{v}^{T}\right]^{T} \otimes\left(q_{2} \otimes q_{1}\right)^{*},
\end{aligned}
$$

where $q_{e q}=q_{2} \otimes q_{1}$ represents the equivalent rotation.

Note that unit quaternion is a double cover of $\mathbb{S O}(3)$ since $q$ and $-q$ represent the same $3 \mathrm{D}$ rotation. Therefore, extra attention is needed when taking quaternions as the inputs of our model. The simplest solution to reducing this ambiguity is to keep the real part of the quaternion positive. Please refer to [49], [50] for more details on unit quaternion and 3D rotation.

\subsection{Quaternion product units}

Given a sequence of $3 \mathrm{D}$ rotations, which is represented as a sequence of quaternions $\boldsymbol{q}=\left\{q_{i}\right\}_{i=1}^{N}$, we can derive an equivalent rotation (quaternion) based on the chain rule in (6):

$$
q_{e q}=q_{1} \otimes q_{2} \otimes \cdots \otimes q_{N}=\bigotimes_{i=1}^{N} q_{i}
$$

where the $q_{e q}$ corresponds to the accumulation of the rotations $\left\{q_{i}\right\}_{i=1}^{N}$ in the reverse order of their subscripts. This process provides an operation to fuse multiple rotations, whose output is closed in $\mathbb{S O}(3)$.

From the viewpoint of neural networks, the fusion of rotations in (7) is analogous to the fusion of vectors achieved by the neuron of the multi-layer perceptron (MLP) [51]. Inspired by this analogy, we propose our quaternion product unit (QPU), which achieves a neuron architecture to fuse rotations. In particular, the neuron in an MLP model can be represented as a weighted summation unit $f: \mathbb{R}^{N} \mapsto \mathbb{R}$, i.e., $y=f(\boldsymbol{x})=\sigma\left(\sum_{i=1}^{N} w_{i} x_{i}+b\right)$, where $\left\{w_{i}\right\}_{i=1}^{N}$ and $b$ are learnable parameters, $\boldsymbol{x}=\left\{x_{i} \in \mathbb{R}\right\}_{i=1}^{N}$ are inputs, $\sigma(\cdot)$ is a nonlinear activation function, and $y \in \mathbb{R}$ is the output signal. Similarly, our QPU is a computational unit mapping from $\mathbb{S O}(3)^{N}$ to $\mathbb{S O}(3)$. It extends the chain of Hamilton products in (7) by introducing learnable parameters.

Specifically, given $N$ unit quaternions $\left\{q_{i}=\left[s_{i}, \boldsymbol{v}_{i}^{T}\right]^{T}\right\}_{i=1}^{N}$ that represent $N$ 3D rotations, we define a weighted chain of Hamilton products as:

$$
y=\bigotimes_{i=1}^{N} q_{i}^{w_{i}}=q_{1}^{w_{1}} \otimes q_{2}^{w_{2}} \otimes \cdots \otimes q_{N}^{w_{N}} .
$$

Here, $q^{w}$ represents the power of the quaternion $q=[s, \boldsymbol{v}]$ with a scalar $w$. It is first proposed in [49] and is defined as

$$
q^{w}:=\left\{\begin{array}{l}
{\left[\cos \left(w \frac{\theta}{2}\right), \boldsymbol{u}^{T} \sin \left(w \frac{\theta}{2}\right)\right]^{T}, \quad \boldsymbol{v} \neq \mathbf{0},} \\
{\left[1, \mathbf{0}^{T}\right]^{T}, \text { otherwise, }}
\end{array}\right.
$$

where $\theta=2 \arccos (s)$ corresponds to the rotation angle. Therefore, the power operation in (9) actually scales the rotation angle while keeps the rotation axis unchanged. If we further consider a bias $b$ imposed on the $\frac{\theta}{2}$, we can define a linear transform of the rotation angle:

qpow $(q ; w, b)=\left[\cos \left(w\left(\frac{\theta}{2}+b\right)\right), \frac{\boldsymbol{v}^{T}}{\|\boldsymbol{v}\|_{2}} \sin \left(w\left(\frac{\theta}{2}+b\right)\right)\right]^{T}$,

where the scaling weight $w$ and the bias $b$ can be explained as the multiplicative perturbation and the additive perturbation of the rotation angle $\theta$, respectively.

In summary, we define the QPU as

$$
\operatorname{QPU}\left(\left\{q_{i}\right\}_{i=1}^{N} ;\left\{w_{i}\right\}_{i=1}^{N}, b\right)=\bigotimes_{i=1}^{N} \operatorname{qpow}\left(q_{i} ; w_{i}, b\right),
$$

where the weights $\left\{w_{i}\right\}_{i=1}^{N}$ and the bias $b$ are learnable parameters. According to the definition in (11), the QPU corresponds to applying linear transforms to the input rotation angles and accumulating the transformed rotations sequentially. Obviously, the output of the QPU is still a quaternion representing the accumulated rotation, so it guarantees the closure of $\mathbb{S O}(3)$.

\subsection{Disentangled rotation-invariance and -equivariance}

As aforementioned, an ideal representation model of 3D structured data should be invariant to the redundant rotations imposed randomly on each data while equivariant to the informative rotations caused by the structural changes within each data. Mathematically, we denote $\mathcal{X}$ as the ambient space of 3D structured data. For an arbitrary rotation $R \in \mathbb{S O}(3)$, a function $f: \mathcal{X} \mapsto \mathcal{Z}$, where $\mathcal{Z}$ is the latent representation space, is rotation-invariant if the following equation holds:

$$
f(R(\boldsymbol{x}))=f(\boldsymbol{x}), \forall \boldsymbol{x} \in \mathcal{X}, R \in \mathbb{S O}(3) .
$$

On the other hand, a function $f: \mathcal{X} \mapsto \mathcal{X}$ is rotation-equivariant if the following equation holds:

$$
f(R(\boldsymbol{x}))=R(f(\boldsymbol{x})), \forall \boldsymbol{x} \in \mathcal{X}, R \in \mathbb{S} \mathbb{O}(3) .
$$


Different from most existing representation methods, which focus on either the rotation-invariance or the rotation-equivariance, our QPU disentangles these two properties explicitly as two parts of its output, which achieves them jointly in a unified algorithmic framework. Specifically, we have the following proposition.

Proposition 1. The $Q P U$ in (11) outputs a quaternion containing a rotation-invariant real part and a rotation-equivariant imaginary part.

Proof. This proposition is followed directly by the property of the Hamilton product. Given two quaternions $\left[s_{1}, \boldsymbol{v}_{1}^{T}\right]^{T}$ and $\left[s_{2}, \boldsymbol{v}_{2}^{T}\right]^{T}$, we apply a Hamilton product, i.e., $\left[s_{1}, \boldsymbol{v}_{1}^{T}\right]^{T} \otimes$ $\left[s_{2}, \boldsymbol{v}_{1}^{T}\right]^{T}=\left[s_{o}, \boldsymbol{v}_{o}^{T}\right]^{T}$. Applying a rotation $R$ on the vector parts, since $\left\langle R\left(\boldsymbol{v}_{1}\right), R\left(\boldsymbol{v}_{2}\right)\right\rangle=\left\langle\boldsymbol{v}_{1}, \boldsymbol{v}_{2}\right\rangle$ and $R\left(\boldsymbol{v}_{1}\right) \times R\left(\boldsymbol{v}_{2}\right)=$ $R\left(\boldsymbol{v}_{1} \times \boldsymbol{v}_{2}\right)$, we have

$$
\begin{aligned}
& {\left[\begin{array}{c}
s_{1} \\
R\left(\boldsymbol{v}_{1}\right)
\end{array}\right] \otimes\left[\begin{array}{c}
s_{2} \\
R\left(\boldsymbol{v}_{2}\right)
\end{array}\right] } \\
= & {\left[\begin{array}{c}
s_{1} s_{2}-\left\langle R\left(\boldsymbol{v}_{1}\right), R\left(\boldsymbol{v}_{2}\right)\right\rangle \\
R\left(\boldsymbol{v}_{1}\right) \times R\left(\boldsymbol{v}_{2}\right)+s_{1} R\left(\boldsymbol{v}_{2}\right)+s_{2} R\left(\boldsymbol{v}_{1}\right)
\end{array}\right] } \\
= & {\left[\begin{array}{c}
s_{1} s_{2}-\left\langle\boldsymbol{v}_{1}, \boldsymbol{v}_{2}\right\rangle \\
R\left(\boldsymbol{v}_{1} \times \boldsymbol{v}_{2}+s_{1} \boldsymbol{v}_{2}+s_{2} \boldsymbol{v}_{1}\right)
\end{array}\right]=\left[\begin{array}{c}
s_{o} \\
R\left(\boldsymbol{v}_{o}\right)
\end{array}\right] . }
\end{aligned}
$$

Thus, the Hamilton product of two quaternions gives a rotationinvariant real part and a rotation-equivariant imaginary part. The same property holds for the chain of weighted Hamilton products.

The proof above indicates that the intrinsic property and the group law of $\mathbb{S O}(3)$ naturally provide both the rotation-invariance and the rotation-equivariance. Without forced tricks or handcrafted representation strategies, the principled design of QPU makes it flexible for a wide range of applications with different requirements on rotation robustness. Fig. 2 further visualizes the property of our QPU. Given a QPU, we feed it with a human skeleton and its rotated version, respectively, in which each skeleton is represented as a set of 3D rotations between adjacent bones. Their outputs verify the above proposition - the imaginary parts of their outputs inherit the rotation discrepancy between them while the real parts of their outputs are the same with each other.

\section{EFFICIENT IMPLEMENTATIONS}

According to (11), a QPU actually is a composite function of quaternions, which consists of $N$ individual "qpow" functions and a chain of their Hamilton products. When applying this neuron model in practice, we need to overcome two computational issues. Firstly, we need to accelerate the feed-forward computation and the backpropagation of QPU, especially reducing the computational complexity of the chained Hamilton products. Secondly, we need to make the QPU and its computation compatible with most existing real-valued neural networks and most 3D application scenarios. In this section, we introduce the implementation of QPU in detail, including the backpropagation of QPU, its acceleration, and its compatibility with various models and data formats.

\subsection{Backpropagation of QPU}

For a QPU, whose inputs are $N$ quaternions $\left\{q_{i}\right\}_{i=1}^{N}$ and parameters are $\left\{w_{i}\right\}_{i=1}^{N}$ and $b$, we are interested in its gradients with respect to its inputs and parameters, respectively. When computing the gradients, we need to first compute the gradients of the "qpow" functions and then apply the backpropagation of the gradients

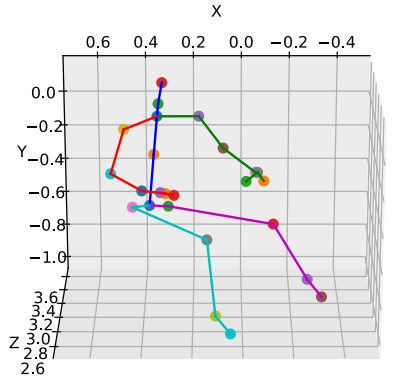

(a) Original

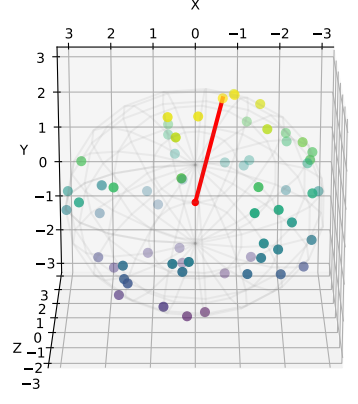

(c) $\boldsymbol{v}_{o}$ corresponding to (a)

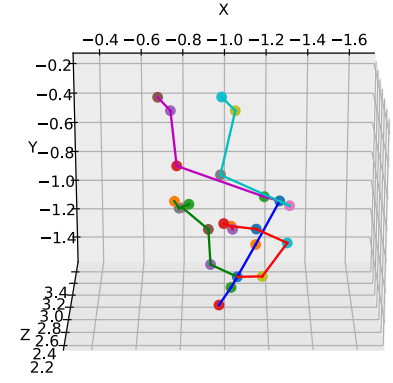

(b) Rotated

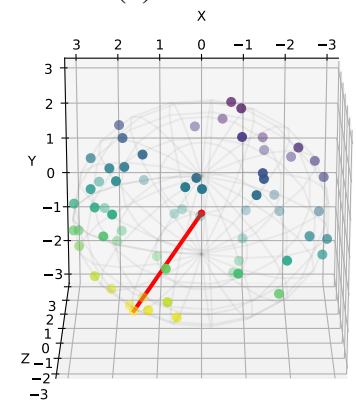

(d) $\boldsymbol{v}_{o}$ corresponding to (b)

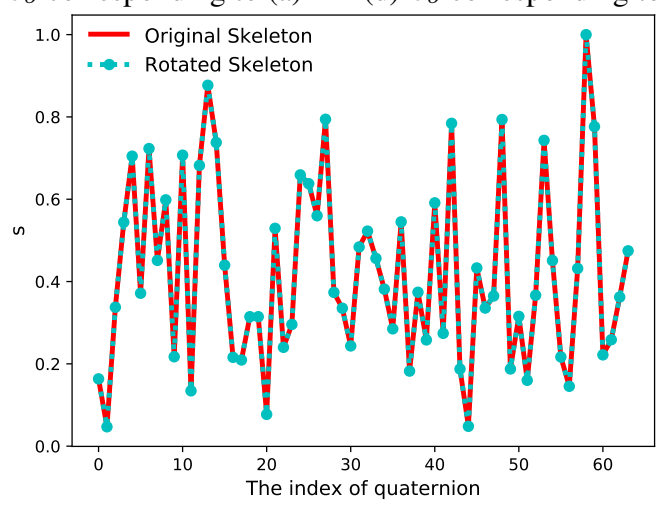

(e) $s_{o}$ 's

Fig. 2: An illustration of the rotation-invariance and the rotationequivariance of QPU. For each skeleton, we represent the relative rotations between its joints as unit quaternions and pass them through a QPU. To visualize the rotation discrepancy, for a $\boldsymbol{v}_{o}$ in (c) and the corresponding $\boldsymbol{v}_{o}$ in (d), we connect each of them (normalized to a sphere) with the origin via a red line.

through the chain of Hamilton products. The first step is relatively easy, whose derivation has been provided in [49]. Specifically, given a quaternion $q=\left[s, \boldsymbol{v}^{T}\right]^{T}$, where $\boldsymbol{v}=\left[v_{1}, v_{2}, v_{3}\right]^{T}$, we denote each qpow $(q ; w, b)$ as a quaternion $p$ for convenience and obtain the following partial derivations:

$$
\begin{aligned}
& \frac{\partial p}{\partial w}=\left[-\theta \sin \omega \theta, v_{1} \theta \mathcal{T}, v_{2} \theta \mathcal{T}, v_{3} \theta \mathcal{T}\right]^{T} \\
& \frac{\partial p}{\partial b}=\left[-\omega \sin \omega \theta, v_{1} \omega \mathcal{T}, v_{2} \omega \mathcal{T}, v_{3} \omega \mathcal{T}\right]^{T} \\
& \frac{\partial p}{\partial q}=\left[\begin{array}{cccc}
\omega \mathcal{I} & 0 & 0 & 0 \\
\frac{-v_{1} \omega}{V} \mathcal{T} & \frac{v_{2}^{2}+v_{3}^{2}}{V^{2}} \mathcal{I} & \frac{-v_{1} v_{2}}{V^{2}} \mathcal{I} & \frac{-v_{1} v_{3}}{V^{2}} \mathcal{I} \\
\frac{-v_{2} \omega}{V} \mathcal{T} & \frac{-v_{1} v_{2}}{V^{2}} \mathcal{I} & \frac{v_{1}^{2}+v_{3}^{2}}{V^{2}} \mathcal{I} & \frac{-v_{2} v_{3}}{V^{2}} \mathcal{I} \\
\frac{-v_{3} \omega}{V} \mathcal{T} & \frac{-v_{1} v_{3}}{V^{2}} \mathcal{I} & \frac{-v_{2} v_{3}}{V^{2}} \mathcal{I} & \frac{v_{1}^{2}+v_{2}^{2}}{V^{2}} \mathcal{I}
\end{array}\right]
\end{aligned}
$$

where $V=\sqrt{1-s^{2}}, \theta=\arccos (s)+b$, and $[\mathcal{T}, \mathcal{I}]=$ $\left[\frac{\cos (\omega \theta)}{V}, \frac{\sin (\omega \theta)}{V}\right]$. Note that the output of arccos contains an 
infinite gradient at \pm 1 . In practice, we solve this problem by clamping the input scalar part $s$ between $-1+\epsilon$ and $1-\epsilon$, where $\epsilon$ is a small number.

The second step is complicated and time-consuming, which is the main bottleneck of QPU in practical applications. The QPU takes $N$ quaternions $\left\{p_{i}=\operatorname{qpow}\left(q_{i} ; w_{i}, b\right)\right\}_{i=1}^{N}$ as its input. Denote the chained Hamilton products of $\left\{p_{i}\right\}_{i=1}^{N}$ (i.e., the output of the QPU) as $y=\bigotimes_{i=1}^{N} p_{i}$. When imposing a perturbation on the $i$-th quaternion, i.e., $\widetilde{p}_{i}=p_{i}+\partial p_{i}$, the chain of the Hamilton products becomes

$$
\begin{aligned}
y+\partial y & =p_{1} \otimes \cdots \otimes\left(p_{i}+\partial p_{i}\right) \otimes \cdots \otimes p_{N} \\
& =B_{i} \otimes\left(p_{i}+\partial p_{i}\right) \otimes A_{i} \\
& =M_{L}\left(B_{i}\right) M_{R}\left(A_{i}\right)\left(p_{i}+\partial p_{i}\right) \\
& =y+M_{L}\left(B_{i}\right) M_{R}\left(A_{i}\right) \partial p_{i},
\end{aligned}
$$

where $M_{L}$ and $M_{R}$ are matrices defined in (3), $B_{i}=\bigotimes_{j<i} p_{j}$ and $A_{i}=\bigotimes_{j>i} p_{j}$ are the chains of the Hamilton products before and after the $i$-th quaternion, respectively. The third equation in (16) is derived from (2). Accordingly, we can get the partial derivative of the chained Hamilton products to $p_{i}$ as

$$
\frac{\partial y}{\partial p_{i}}=M_{R}^{T}\left(A_{i}\right) M_{L}^{T}\left(B_{i}\right)
$$

When applying the QPU as a module of neural network and learning the model based on a scalar loss function $L$, the gradient of $L$ with respect to the QPU's output $y$, which can be computed by the auto grad [52] of Pytorch, is

$$
\begin{aligned}
\partial L & =\left\langle\frac{\partial L}{\partial y}, \partial y\right\rangle \\
& =\left\langle\frac{\partial L}{\partial y}, M_{L}\left(B_{i}\right) M_{R}\left(A_{i}\right) \partial p_{i}\right\rangle \\
& =\left\langle M_{R}^{T}\left(A_{i}\right) M_{L}^{T}\left(B_{i}\right) \frac{\partial L}{\partial y}, \partial p_{i}\right\rangle
\end{aligned}
$$

where $\langle\cdot, \cdot\rangle$ stands for the inner product between vectors and $M^{T}$ stands for the transpose of matrix $M \cdot \frac{\partial L}{\partial y}$ is the gradient of the loss $L$ for the QPU's output, which is provided by the successive module of the QPU. Therefore, the gradient of the loss $L$ for $p_{i}$ is given as

$$
\frac{\partial L}{\partial p_{i}}=M_{R}^{T}\left(A_{i}\right) M_{L}^{T}\left(B_{i}\right) \frac{\partial L}{\partial y} .
$$

In summary, combining (15) with (19), we derive the backpropagation of QPU as follows:

$$
\frac{\partial L}{\partial q_{i}}={\frac{\partial p_{i}}{\partial q_{i}}}^{T} M_{R}^{T}\left(A_{i}\right) M_{L}^{T}\left(B_{i}\right) \frac{\partial L}{\partial y},
$$

and its own parameters' update is achieved by

$$
\begin{aligned}
& \frac{\partial L}{\partial w_{i}}=\frac{\partial p_{i}}{\partial w_{i}} M_{R}^{T}\left(A_{i}\right) M_{L}^{T}\left(B_{i}\right) \frac{\partial L}{\partial y} \\
& \frac{\partial L}{\partial b}=\sum_{i=1}^{N} \frac{\partial p_{i}{ }^{T}}{\partial b} M_{R}^{T}\left(A_{i}\right) M_{L}^{T}\left(B_{i}\right) \frac{\partial L}{\partial y} .
\end{aligned}
$$

This backpropagation step is different from that of the neuron in conventional MLP, whose backpropagation through addition only involves the differential variable instead of other inputs. In contrast, the gradient of a QPU is a joint result of all other inputs to the QPU except the items of the given differential variable.

\subsection{Algorithmic acceleration for fast QPUs}

For a QPU taking $N$ quaternions as its input, when we implement its feed-forward step directly based on (11), its time complexity is $\mathcal{O}(N)$ (for $N-1$ Hamilton products) and consumes $\mathcal{O}(N)$ units of memory (for storing the inputs). In the backpropagation step, however, we need to compute $B_{i}=\bigotimes_{j<i} p_{j}$ and $A_{i}=$ $\bigotimes_{j>i} p_{j}$, respectively, for $i=1, \ldots, N$, and save the gradients accordingly. Therefore, for the backpropagation of the QPU, its space complexity is $\mathcal{O}(N)$ (comparable to that of the feed-forward step), while its time complexity is $\mathcal{O}\left(N^{2}\right)$, which is significantly higher than that of the neuron of $\operatorname{MLP}(i . e ., \mathcal{O}(N)$ ). To make our QPU applicable in practice, we integrate various acceleration strategies and propose a fast version of QPU accordingly.

Strategy 1: Acceleration of backpropagation. For the $B_{i}$ 's and $A_{i}$ 's used in the backpropagation, we can find that $B_{1}=$ $A_{N}=\left[1, \mathbf{0}^{T}\right]^{T}$ and for $i=1, \ldots, N-1$

$$
A_{i}=\bigotimes_{j>i} p_{j}=\left(\bigotimes_{j<i+1} p_{j}\right)^{*} \otimes\left(\bigotimes_{j=1}^{N} p_{j}\right)=B_{i+1}^{*} \otimes y .
$$

Moreover, the $B_{i}$ 's have been calculated (as intermediate quaternions) in the feed-forward step. Therefore, by retaining all the $B_{i}$ 's calculated in the feed-forward computation step, we can avoid redundant computations in the backpropagation step and reduce its time complexity from $\mathcal{O}\left(N^{2}\right)$ to $\mathcal{O}(N)$.

Strategy 2: Acceleration of feed-forward computation. We can apply the multi-thread reduction strategy [53] to accelerate the feed-forward step. In particular, this strategy leverages the associativity of the Hamilton product, reorganizing the chained products with the help of a tree structure, as illustrated in Fig. 3. Here, in order to access each tree node more conveniently, we set the tree to a full binary tree structure and use $\left[1, \mathbf{0}^{T}\right]^{T}$ as dummy nodes. As a result, we can achieve the feed-forward computation in parallel and store the processing results of each thread in the tree. Accordingly, the time complexity of the feed-forward computation is reduced from $\mathcal{O}(N)$ to $\mathcal{O}(\log N)$.

Strategy 3: Acceleration of both backpropagation and feed-forward computation. It should be noted that the above two acceleration strategies cannot be applied simultaneously in general because $i$ ) the Hamilton product is non-commutative, and ii) the multi-thread reduction strategy in Fig. 3 does not compute all $B_{i}$ 's one-by-one. In this work, we suppress this conflict by a novel method, which is inspired by the properties of the Hamilton product.

Firstly, given the sequence of the quaternions $\left\{p_{i}\right\}_{i=1}^{N}$, and we sort them in ascending order from left to right. The binary tree structure in Fig. 3 indicates that on the backward way from the root to the leaf $p_{i}$ (i.e., the path consisting of dotted arrows), all the sibling nodes (i.e., the green $\&$ blue nodes) of the passing node (i.e., the yellow nodes) exactly contain all the intermediate results required for calculating the partial derivative of $p_{i}$. Moreover, leveraging the binary tree structure, we have the following three properties:

- Property 1 (Coverage). Its left sibling nodes involve all the $p_{j}$ 's with $j<i$, while its right sibling nodes involve all the $p_{j}$ 's with $j>i$.

- Property 2 (Sorting w.r.t. depth). for the backward way from the root to the leaf $p_{i}$, with the increase of depth from 0 to $\lceil\log N\rceil$, the quaternions involved in its left sibling nodes are sorted in ascending order, while those involved in its right sibling nodes are organized in descending order. 
- Property 3 (Mutual exclusion). For the backward way from the root to the leaf $p_{i}$, each of its passing nodes only owns one sibling node, on either the left-side or the right-side of the backward way.

Secondly, for arbitrary three adjacency quaternions $p_{i-1}, p_{i}$ and $p_{i+1}$ in a chain, we have

$$
\begin{aligned}
& \underbrace{\left(p_{i-1} \otimes p_{i}\right) \otimes p_{i+1}=p_{i-1} \otimes\left(p_{i} \otimes p_{i+1}\right)}_{\text {(Associativity) }} \\
\Leftrightarrow & \left(M_{L}\left(p_{i-1}\right) p_{i}\right) \otimes p_{i+1}=p_{i-1} \otimes\left(M_{R}\left(p_{i+1}\right) p_{i}\right) \\
\Leftrightarrow & M_{R}\left(p_{i+1}\right) M_{L}\left(p_{i-1}\right) p_{i}=M_{L}\left(p_{i-1}\right) M_{R}\left(p_{i+1}\right) p_{i} \\
\Leftrightarrow & \underbrace{M_{R}\left(p_{i+1}\right) M_{L}\left(p_{i-1}\right)=M_{L}\left(p_{i-1}\right) M_{R}\left(p_{i+1}\right)}_{\text {(Limited commutability) }} .
\end{aligned}
$$

The last line of (23) indicates the limited commutability of the Hamilton product: for the center quaternion $p_{i}$, the right-side matrix of its right-side quaternion and the left-side matrix of its left-side quaternion are exchangeable.

Taking the tree structure and the above property into account, we can find that although the left/right sibling nodes appear in an order controlled by the tree structure rather than sequentially, we only need to convert them into the corresponding left/right matrices and multiply them directly when calculating each partial derivative $\frac{\partial y}{\partial p_{i}}$. In particular, for the backward way from the root to the leaf $p_{i}$, we denote $S_{i, j}^{L / R}$ as the left/right sibling nodes at the $j$-th level of the tree, each of which is set to $\left[1, \mathbf{0}^{T}\right]^{T}$ when it does not exit. Accordingly, we can derive $\left(\frac{\partial y}{\partial p_{i}}\right)^{T}$ as

$$
\begin{aligned}
\left(\frac{\partial y}{\partial p_{i}}\right)^{T} & =M_{L}\left(B_{i}\right) M_{R}\left(A_{i}\right) \\
& =\underbrace{\prod_{k=1}^{i-1} M_{L}\left(p_{k}\right)}_{\text {Ascending-order indexing Descending-order indexing }} \underbrace{\prod_{k=N}^{i+1} M_{R}\left(p_{k}\right)} \\
& =\prod_{j=0}^{\log N\rceil} M_{L}\left(S_{i, j}^{L}\right) \prod_{j=0}^{\text {log } N\rceil} M_{R}\left(S_{i, j}^{R}\right) \\
& =\prod_{j=0}^{\lceil\log N\rceil} M_{L}\left(S_{i, j}^{L}\right) M_{R}\left(S_{i, j}^{R}\right) \\
& =\prod_{j=0}^{\log N\rceil} M_{L / R}\left(S_{i, j}^{L / R}\right) .
\end{aligned}
$$

In (24), the second equation is based on the definitions of $B_{i}$ and $A_{i}$ and the rule of Hamilton product; The third equation is based on the property 1 and property 2 of the tree structure; The fourth equation is derived by applying the limited commutability shown in (23) $\lceil\log N\rceil$ times; The final equation is based on the property 3 of the tree structure $-S_{i, j}^{L}$ and $S_{i, j}^{R}$ are mutually exclusive, so that $M_{L}\left(S_{i, j}^{L}\right) M_{R}\left(S_{i, j}^{R}\right)$ equals to either $M_{L}\left(S_{i, j}^{L}\right)$ or $M_{R}\left(S_{i, j}^{R}\right)$. In other words, we just need $\lceil\log N\rceil$ Hamilton products when computing the gradient of $B_{i}$.

Note that the indexing of the passing nodes and their sibling nodes is simple and efficient. As shown in Fig. 3, starting from the root node, we index the nodes in the tree with the increase of depth. For the nodes with the same depth, we index them from left to right. Consider the binary representations of the indices. Accordingly, given a backward way, we can 1) index the passing node with depth $j-1$ by applying 1-bit logical right-shifting to the index of the passing node with depth $j$; and 2) index the sibling node with depth $j$ by applying the XOR between 1 and the index of the passing node with depth $j$. Therefore, given the index of the target node $p_{i}$, we can find the indices of the nodes used to compute $\frac{\partial y}{\partial p_{i}}$ by $2\lceil\log N\rceil$ bit operations, which is very fast.

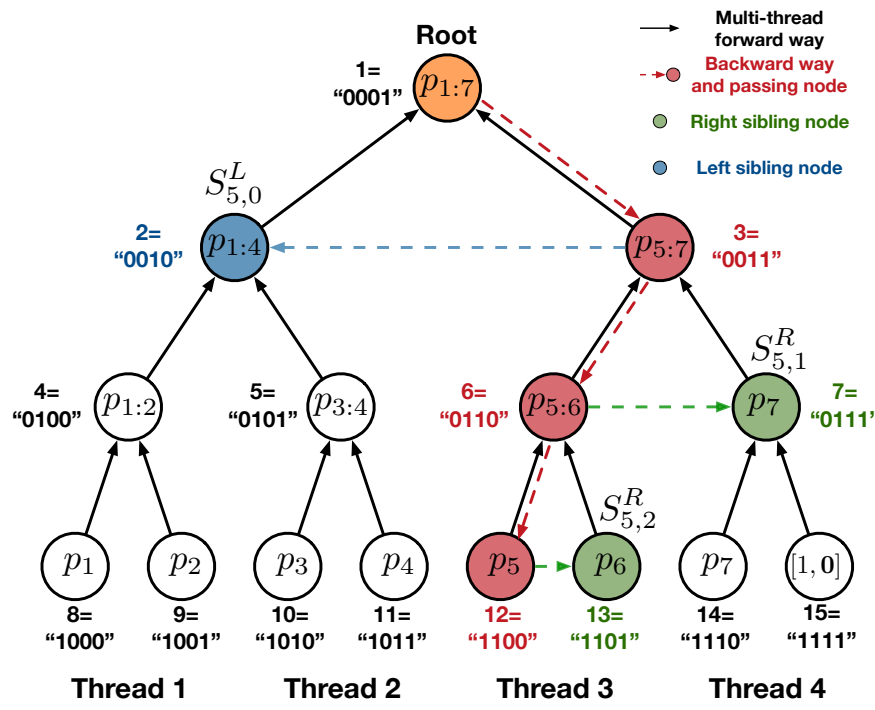

Fig. 3: An illustration of the tree structure stored by a QPU when $N=7$. Given a backward way, its passing nodes are indicated by the red nodes at different depths, and at each depth, the left/right sibling node of the passing node is indicated by blue/green nodes. Each node is associated with an index, which is also represented in a binary format. Given a target node $\left(i . e ., p_{5}\right)$, we can construct its backward way by indexing the corresponding passing nodes and sibling nodes. Accordingly, the gradient of the QPU to the target node can be computed by (24).

With the help of this acceleration strategy, for a QPU, we can keep the $\mathcal{O}(\log N)$ complexity for its feed-forward computation. At the same time, the time complexity of computing each $B_{i}$ and its gradient becomes $\mathcal{O}(\log N)$ as well, which reduces the time complexity of the backpropagation to $\mathcal{O}(N \log N)$. In other words, strategy 3 achieves a trade-off between the former two strategies.

\subsection{Accelerating memory access for fast QPUs}

In addition to the algorithm and the data structure, memory access is also an important performance bottleneck in large-scale parallel computing. Modern computers have established a multi-level caching mechanism to reduce the impact of memory access on computing performance by using its temporal and spatial locality. When accessing the data unit, a computer will query the existence of the data in order from the high-speed cache to the low-speed cache. If data exists, the computer will move it and its surrounding to the upper-level cache, thereby reducing the speed of accessing adjacent data. Therefore, when accessing a batch of data, the closer they are in the memory, the less time we cost.

Inspired by the above analysis, we organize the storage structure of our data by referring to their calculation order. As shown in Fig. 3, the quaternions used by each thread in the feedforward computation happen to be adjacent quaternions on the memory, which own good locality and can further demonstrate the reliability of the proposed tree structure. In addition, given $N$ quaternions, we can store them as an array, whose format is either $N \times 4$ or $4 \times N$. For arbitrary two adjacent quaternions $q_{i}$ and $q_{i+1}$, applying the former format makes their relative memory coordinates adjacent as well, i.e., $\{4 i+k, 4(i+1)+k \mid k \in[0,3]\}$. On the contrary, when applying the later format, their relative 


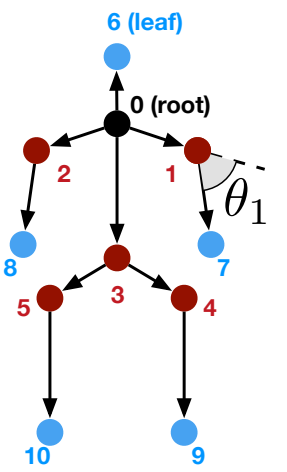

(a) Skeleton

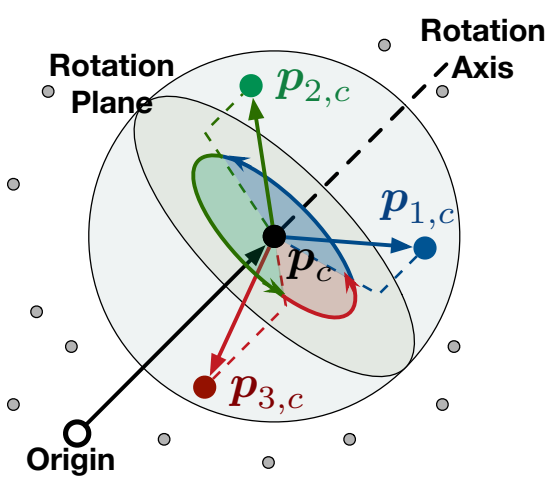

(b) Point cloud
Fig. 4: (a) An illustration of the representation of skeleton data. The $\theta_{1}$ associated with the node " 1 " indicates the rotation from the edge $0 \rightarrow 1$ to the edge $1 \rightarrow 7$. (b) An illustration of the neighborhood of a centroid. The small gray points indicate the points out of the range. The black point represents the centroid, and the color points are its neighbors. The color sector areas on the rotation plane represents the angles used to sort the neighbor points. The arrows associated with the sector areas indicate the order of the neighbor points.

memory coordinates become $\{k N+i, k N+i+1 \mid k \in[0,3]\}$, and accordingly, the two quaternions are far from each other in the memory. Therefore, in this work, we store the input quaternions of a QPU in the $N \times 4$ format, such that we can leverage the compact memory locations of the quaternions to accelerate the memory access of QPU.

\section{Building Neural Networks via Fast QPUs}

Our fast QPU leads to a new neuron model for 3D rotations, which achieves a fusion operation closed in $\mathbb{S O}(3)$. Taking it as a basic module, we can develop various quaternion neural networks (QNNs) for modeling skeletons and point clouds, respectively. Additionally, we consider the compatibility of the QNNs with existing real-valued models and make efforts to solve the permutation-sensitivity issue caused by the non-commutativity of the Hamilton product.

\subsection{Representing 3D data as 3D rotations}

As shown in Fig. 4a, given a skeleton, we can represent it as a tree in which the nodes are indexed in advance and the root is predefined. All the leaves (i.e., the blue nodes) and the root (i.e., the black nodes) are associated with $\left[1,0^{T}\right]^{T}$. For each of the other nodes (i.e., the red nodes), we represent the rotation from its preceding edge and succeeding edge as a unit quaternion, in which the rotation axis and the rotation angle can be derived by (1). Accordingly, a skeleton can be formulated as a sequence of quaternions.

Representing a point cloud is more challenging than representing a skeleton because the points are unordered while our QPU requires ordered inputs (due to the non-commutativity of the Hamilton product). Therefore, we need to organize the points first before defining the $3 \mathrm{D}$ rotations between them. In this work, we leverage the sampling-and-grouping method used in the Pointnet++ [23] to achieve this aim. Given a point cloud, we first estimate the global center of its point as the origin of a coordinate system and then sample 256 grid centroids uniformly in the system. As shown in Fig. 4b, for the $c$-th centroid, denoted as $\boldsymbol{p}_{c}$, we search 32 neighbor points in a ball with a radius $R=0.4$, i.e., $\left\{\boldsymbol{p}_{n, c}\right\}_{n \in \mathcal{N}_{c}}$, where $\mathcal{N}_{c}$ is the neighborhood of $\boldsymbol{p}_{c}$ and $\left|\mathcal{N}_{c}\right|=32$. Accordingly, $\boldsymbol{p}_{n, c}=\boldsymbol{p}_{n}-\boldsymbol{p}_{c}$ represents the local 3D coordinates of each neighbor point with respect to its centroid. For each $\boldsymbol{p}_{n, c}$, we empirically define a unit quaternion, i.e., $q_{n, c}=\left[\cos \left(\theta_{n, c}\right), \sin \left(\theta_{n, c}\right) \boldsymbol{u}_{n, c}^{T}\right]^{T}$, as its feature, where $\boldsymbol{u}_{n, c}=\frac{\boldsymbol{p}_{n, c}}{\left\|\boldsymbol{p}_{n, c}\right\|_{2}}$ is the rotation axis and $\theta_{n, c}=\frac{\pi\left\|\boldsymbol{p}_{n, c}\right\|_{2}}{2 R}$ is the rotation angle. Because the rotation angle is proportional to $\left\|\boldsymbol{p}_{n, c}\right\|_{2}$, this definition preserves the length information of $\boldsymbol{p}_{n, c}$, and the feature works well in the following experiments.

Given the $q_{n, c}$ 's, we consider determining their order by the following three methods:

- Rotation-based Sorting [11]. As shown in Fig. 4b, the vector from the origin to the centroid defines a rotation axis. Projecting the $\boldsymbol{p}_{n, c}$ 's to the rotation plane that is orthogonal to the rotation axis, we can derive the rotation angle between each adjacent projection. Accordingly, starting from the $q_{n, c}$ whose $\boldsymbol{p}_{n, c}$ is the closest point to the rotation plane, we can sort the $q_{n, c}$ 's in a clockwise rotation order (when looking at the plane along the direction of $\boldsymbol{p}_{c}$ ).

- Angle-based Sorting. Based on $\boldsymbol{p}_{c}$, we can also permute the $q_{n, c}$ 's in ascending order of the angles between $\boldsymbol{p}_{c}$ and $\boldsymbol{p}_{n, c}$.

- Distance-based Sorting. Finally, we can permute $q_{n, c}$ 's in ascending order of the lengths $\left\|\boldsymbol{p}_{n, c}\right\|_{2}$ 's.

Applying one of the above sorting methods, we obtain a sequence of quaternions for each centroid. In summary, the point cloud is represented as a set of such sequences.

\subsection{Typical QNNs built by fast QPUs}

Given the sequences of quaternions generated by the abovepreprocessing steps, we can build various quaternion neural networks based on our fast QPUs and represent the sequences accordingly. Below, we introduce some typical QNNs built in this work.

Quaternion multi-layer perceptron (QMLP). A set of QPUs receiving the same input quaternions leads to a fully-connected (FC) layer. The parameters of the QPU-based FC layer include the weights and the bias used in the QPUs, which can be initialized by the Xavier uniform initialization [54]. We can connect multiple QPU-based FC layers sequentially because the outputs of the QPUs are still unit quaternions. Accordingly, we can build a quaternion multi-layer perceptron (QMLP) by stacking multiple QPU-based FC layers, as shown in Fig. 5a. Different from the neuron used in conventional real-valued MLP models, the QPU itself is nonlinear so that we don't need to introduce additional nonlinear activation functions in the QMLP model.

Quaternion message passing (QMP). In practice, a complicated 3D skeleton may be represented as a directed graph. As shown in Fig. 5b, each node of such a skeleton may own multiple indexed preceding nodes and succeeding nodes, and all the nodes are represented as 3D rotations by the preprocessing step introduced in Section 5.1.

Focusing on the 3D rotations associated with a directed graph structure, we propose a quaternion message passing (QMP) layer to merge their information. In particular, for each node, its corresponding 3D rotation is represented as a quaternion $q$. Denote $\left\{q_{i}^{\text {in }}\right\}_{i=1}^{N}$ as the quaternions of its preceding nodes and $\left\{q_{j}^{\text {out }}\right\}_{j=1}^{M}$ 


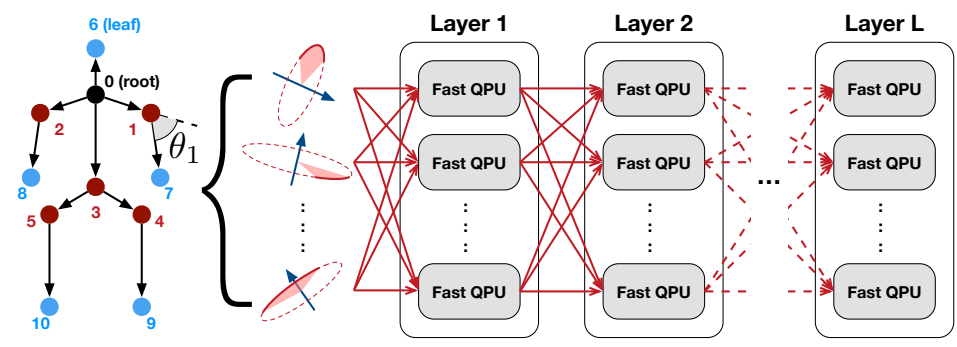

(a) Quaternion multi-layer perceptron (QMLP)

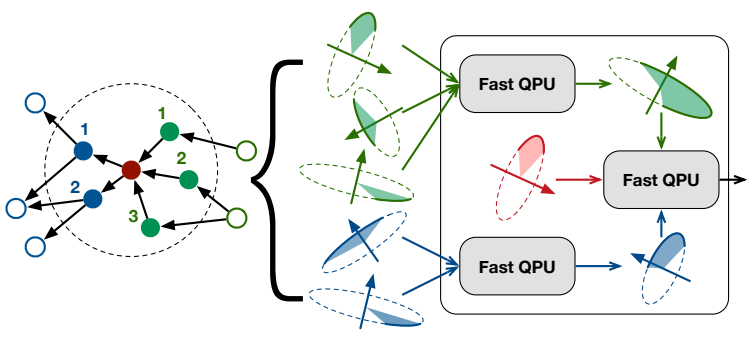

(b) Quaternion message passing (QMP)

Fig. 5: Typical quaternion neural network layers constructed by our fast QPUs.

as the quaternions of its succeeding nodes. Our QMP layer merges the quaternions via

$$
\operatorname{QPU}\left(\left\{q, \operatorname{QPU}\left(\left\{q_{i}^{\text {in }}\right\}_{i=1}^{N}\right), \operatorname{QPU}\left(\left\{q_{j}^{\text {out }}\right\}_{j=1}^{M}\right)\right\}\right) .
$$

We illustrate this operation in Fig. 5b. Here, the proposed QMP defines a hierarchical structure of three fast QPUs. The two fast QPUs in the first layer merge the quaternions of the preceding nodes and those of the succeeding nodes, respectively. Their outputs, together with the quaternion of the center node, pass through the third fast QPU and lead to the merging result. By stacking multiple QMP layers, we can obtain a quaternion graph neural network for skeleton representation.

Quaternion set abstraction layer (QSA). Combining the preprocessing step for point clouds in Section 5.1 with the above QMLP, we obtain a quaternion set abstraction (QSA) layer. This layer imitates the conventional set abstraction layer used in the PointNet-based models [13], [23]. In particular, the QSA layer first samples centroid points, and then sorts their neighbors, and finally, pass the sorted neighbors to a QMLP module. In the following experiments, we will leverage the QSA layer and demonstrate its feasibility.

Quaternion relationship convolution (QRSConv). Relationship convolution (RSConv) [26] is a classic layer for convolutionbased point cloud modeling. It defines a continuous convolution kernel in the neighborhood of each centroid and implements the continuous convolution kernel as an MLP network. Inspired by this module, we propose a quaternion relationship convolution (QRSConv) layer accordingly. Specifically, given a centroid $\boldsymbol{p}_{c}$, its neighbors $\left\{\boldsymbol{p}_{n}\right\}_{n=1}^{\left|\mathcal{N}_{c}\right|}$, and the local coordinates of the neighbors $\left\{\boldsymbol{p}_{n, c}=\boldsymbol{p}_{n}-\boldsymbol{p}_{c}\right\}_{n=1}^{\left|\mathcal{N}_{c}\right|}$, we define the relational information between the centroid and its each neighbor as a vector $h_{n, c}=\left[\left\|\boldsymbol{p}_{n, c}\right\|_{2}, \arccos \frac{\left\langle\boldsymbol{p}_{n}, \boldsymbol{p}_{c}\right\rangle}{\left\|\boldsymbol{p}_{n}\right\|\left\|\boldsymbol{p}_{c}\right\|}\right]$, which contains the distance and the angle between the two points, respectively. Taking the quaternion and the relational information of each neighbor point as input, our QRSConv is defined as

$$
\begin{aligned}
& \operatorname{QRSConv}_{f}\left(\left\{h_{n, c}, q_{n, c}\right\}_{n=1}^{\left|\mathcal{N}_{c}\right|}\right)= \\
& g\left(\operatorname{MLP}\left(\left\{h_{n, c}\right\}_{n=1}^{\left|\mathcal{N}_{c}\right|}\right) \odot f\left(\operatorname{QMLP}\left(\left\{q_{n, c}\right\}_{n=1}^{\left|\mathcal{N}_{c}\right|}\right)\right)\right),
\end{aligned}
$$

where $g(\cdot)=\operatorname{MLP}(\operatorname{MaxPool}(\cdot))$ is a stacking of a maxpooling layer and an MLP, “ $\odot$ " represents the Hardmard product. $f: \mathbb{H} \mapsto \mathbb{R}^{4}$ is a function converting a quaternion to four real numbers, which can be used as a post-process of our fast QPU when connecting to real-valued models. Suppose that the QMLP in (26) outputs $N$ quaternions, the function $f$ converts them to a $N \times 4$ matrix. $^{2}$ In this work, we implement this function by an angle-axis map (AAM). Specifically, given a unit quaternion $q=\left[s, \boldsymbol{v}^{T}\right]^{T}$, the proposed mapping function is defined as:

$$
f(q):=\operatorname{AngleAxisMap}(q)=\left[\arccos (s), \frac{\boldsymbol{v}^{T}}{\|\boldsymbol{v}\|_{2}}\right]^{T} .
$$

This function maps the quaternions that lie on a non-Euclidean manifold [55], to the Euclidean space. It is better than the logarithmic map used in [6], [14], [29], [37] — the logarithmic map $\log (q)=\left[0, \arccos (s) \frac{\boldsymbol{v}^{T}}{\|\boldsymbol{v}\|_{2}}\right]^{T}$ mixes the angular and axial information, while our map preserves their disentanglement, which is essential to preserve the rotation-invariance (i.e., the $\arccos (s)$ ) and the rotation-equivariance (i.e., the $\frac{\boldsymbol{v}}{\|\boldsymbol{v}\|_{2}}$ ), respectively. Obviously, besides the QMLP, the angle-axis map in (27) can be used for any other QPU-based QNNs.

For an arbitrary QNN, we will use the angle-axis map as the default $f$ in the following experiments. Additionally, we can make the function $f$ more sophisticated and propose some variants of the QNN by modifying the angle-axis map. Given the quaternion $q=\left[s, \boldsymbol{v}^{T}\right]^{T}$ derived by the QNN, we consider the following variants:

- $\mathbf{Q N N} N_{\mathbf{R}}$ sets $f(q)=\arccos (s)$, which purely outputs the rotation-invariant features

- $\mathbf{Q N} \mathbf{N}_{\mathbf{I}}$ sets $f(q)=\frac{\boldsymbol{v}}{\|\boldsymbol{v}\|_{2}}$, which purely outputs the rotationequivarant features.

- $\mathbf{Q N N}_{\mathbf{R I}}$ leverage a special dyadic mapping function. Specifically, given a pair of adjacent inputs of QPU, i.e., $q_{i}=$ $\left[s_{i}, \boldsymbol{v}_{i}^{T}\right]^{T}$ and $q_{i+1}=\left[s_{i+1}, \boldsymbol{v}_{i+1}^{T}\right]^{T}$, we have $f\left(q_{i}, q_{i+1}\right)=$ $\left[\arccos \left(s_{i}\right), \boldsymbol{v}_{i}^{T} \boldsymbol{v}_{i+1}\right]$. The inner product $\boldsymbol{v}_{i}^{T} \boldsymbol{v}_{i+1}$ is rotationinvariant as well, which further enriches the information of the output and preserves its rotation-invariance at the same time.

In the following experiments, we will show that using these variants achieves encouraging performance in different scenarios.

\section{EXPERIMENTS}

In this section, we first verify the rationality of our QPU on a synthetic dataset, and then we apply various QPU-based QNNs to some representative real-world skeleton and point cloud modeling tasks and compare them with state-of-the-art methods. Given a model and a dataset, we train the model on the training set provided by the dataset, but consider the following two testing scenarios:

2. It should be noted that the output of the MLP in (26) can be with arbitrary dimensions. Therefore, we configure the MLP according to the output of the QMLP and the design of the function $f$. 


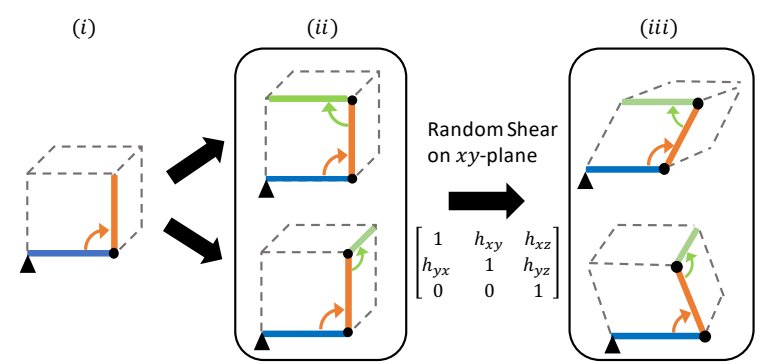

(a) Generation process of CubeEdge.
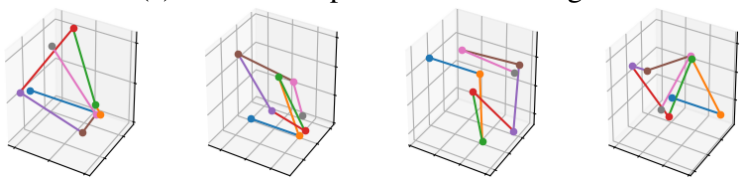

(b) Typical testing samples of CubeEdge with 7 edges

Fig. 6: Illustrations of the CubeEdge dataset. (a) The steps to generate the skeletons: $i$ ) fix the two first edges; $i i$ ) pick the next edge from two possible candidates (doubling the number of classes); $i$ ii) apply random shear on $x y$-plane. The root vertex is marked with a triangle. (b) Samples from the testing set.

- Non-rotated (NR) data: applying the model to the testing set directly, without any random rotations imposed on the testing samples.

- Arbitrarily-rotated (AR) data: imposing additional random rotations on the testing data and applying the model accordingly.

The first scenario aims at evaluating the feature extraction ability of the model, while the second one aims at validating its robustness to rotations quantitatively. Finally, the ablation study of important modules is provided, and we demonstrate the computational efficiency of our fast QPU.

\subsection{Experiments on synthetic data}

We first design a synthetic dataset called "CubeEdge" and propose a simple task to demonstrate the rotation robustness of our QPUbased representation models. The dataset consists of skeletons composed of consecutive edges taken from a 3D cube (a.k.a, a chain of edges). These skeletons are categorized into classes according to their shapes, i.e., the topology of consecutive edges. For each skeleton, we use the rotations between consecutive edges as its feature.

For each skeleton in the dataset, we generate it via the following three steps, as shown in Fig. 6a: $i$ ) We initialize the first two adjacent edges deterministically. ii) We ensure that the consecutive edges cannot be the same edge, so given the ending vertex of the previous edge, we generate the next edge randomly along with either of the two valid directions. Accordingly, each added edge doubles the number of possible shapes. We repeat this step several times to generate a skeleton. iii) We apply random shear transformations to the skeletons and augment the dataset, and split the dataset for training and testing. We generate each skeleton with seven edges so that our dataset consists of 32 different shapes (classes) of skeletons. We use 2,000 samples for training and testing, respectively. For the skeletons in the testing set, we add Gaussian noise $\mathcal{N}\left(0, \sigma^{2}\right)$ to their vertices before shearing, where the standard deviation $\sigma$ controls the level of noise. Fig. $6 \mathrm{~b}$ gives four testing samples. Additional random

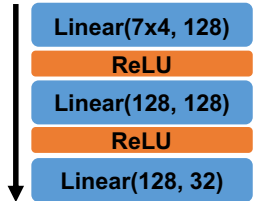

(a) RMLP

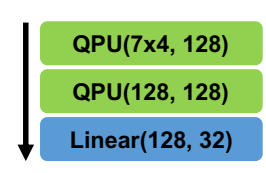

(b) QMLP

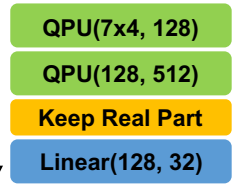

(c) $\mathrm{QMLP}_{\mathrm{R}}$
Fig. 7: The architectures of the models for synthetic data.

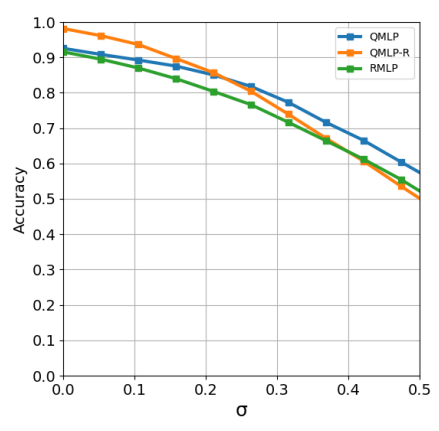

(a) The NR scenario

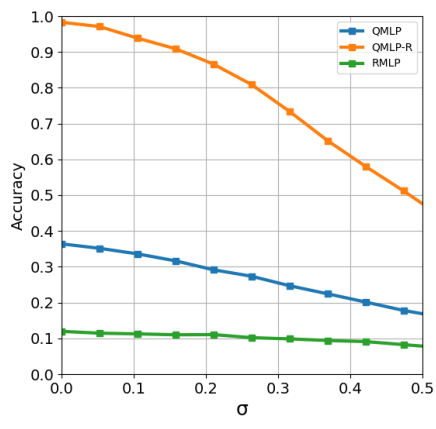

(b) The AR scenario
Fig. 8: Comparisons on testing accuracy.

rotation is applied to the testing set when validating the robustness to rotations.

We train a QMLP model and its variant $\mathbf{Q M L P} \mathbf{P}_{\mathbf{R}}$ to classify the synthetic skeletons and compare them with a standard realvalued MLP (RMLP). For fairness, all three models are implemented with three layers, in which the last layer is unified as a fully connected classifier with the same size as the accepted feature map. The architectures of these three models are shown in Fig. 7. These three models are trained under the same hyperparameter setting. During the training process, we trained the model with 200 as the batch size for 100 epochs, and the learning rate was 0.001. The optimizer used is Adam [56], and its weight decay parameter is set to $10^{-5}$. As aforementioned, we consider both $\mathrm{NR}$ and AR scenarios in the testing phase. For each scenario, we compare these three models on their classification accuracy. Their results with respect to different levels of noise (i.e., $\sigma$ ) are shown in Fig. 8. In both scenarios, our QMLP and QMLP $_{R}$ outperform the RMLP consistently, which demonstrates the superiority of our QPU model. Furthermore, we find that the $\mathrm{QMLP}_{\mathrm{R}}$ is robust to the random rotations imposed on the testing data - it achieves nearly the same accuracy in both scenarios and works better than QMLP in the scenario with random rotations. This phenomenon verifies our claims: $i$ ) the real part of our QPU's output is rotationinvariant indeed; $i i$ ) the disentangled representations of rotationinvariant and rotation-equivariant features could inherently make the network robust to rotations.

\subsection{Verification of acceleration}

Besides the rotation robustness, we also verify the efficiency of the fast QPU achieved by our acceleration strategy. In particular, we consider three QMLP models. The first QMLP consists of a single layer with 2048 QPUs, which maps 256 quaternions to 2048 quaternions. The second QMLP consists of two QPU-based layers, first mapping 256 quaternions to 2048 quaternions and then mapping the 2048 quaternions back to 256 quaternions. The third 
TABLE 1: Comparisons for various implementations on their computational efficiency.

\begin{tabular}{ccccc}
\hline \hline QMLP Architecture & Implementation & Memory (MB) & Forward(ms) & Backward(ms) \\
\hline \multirow{2}{*}{$\mathbb{H}^{256} \mapsto \mathbb{H}^{2048}$} & QPU (AutoGrad) & 6999 & 1.88 & 292.71 \\
& Fast QPU (Strategy 3) & 4519 & 0.32 & 221.59 \\
\hline \multirow{2}{*}{$\mathbb{H}^{256} \mapsto \mathbb{H}^{2048} \mapsto \mathbb{H}^{256}$} & QPU (AutoGrad) & 9267 & 4.36 & 591.44 \\
& Fast QPU (Strategy 3) & 6554 & 0.56 & 464.11 \\
\hline \multirow{2}{*}{$\mathbb{H}^{64} \mapsto\left(\mathbb{H}^{256} \mapsto \mathbb{H}^{256}\right)_{\times 8} \mapsto \mathbb{H}^{64}$} & QPU (AutoGrad) & 2979 & 44.03 & 314.97 \\
& Fast QPU (Strategy 3) & 2689 & 2.56 & 244.96 \\
\hline \hline
\end{tabular}

TABLE 2: Comparisons on testing accuracy (\%) on NTU and FPHA.

\begin{tabular}{lc|cc|cc}
\hline \hline \multirow{2}{*}{ Model } & \#Param. & \multicolumn{2}{|c|}{ NTU } & \multicolumn{2}{c}{ FPHA } \\
& (Million) & NR & AR & NR & AR \\
\hline RMLP-LSTM & 0.691 & 72.92 & 24.67 & 67.13 & 17.39 \\
QMLP-LSTM & 0.609 & 69.72 & 26.60 & $\mathbf{7 6 . 1 7}$ & 24.00 \\
QMLP-LSTM $_{\mathrm{R}}$ & 0.621 & $\mathbf{7 5 . 8 4}$ & $\mathbf{7 5 . 8 4}$ & 68.00 & $\mathbf{6 7 . 8 3}$ \\
\hline AGC-LSTM $^{\text {QAGC-LSTM }}$ & 23.371 & $\mathbf{9 0 . 5 0}$ & 55.17 & 77.22 & 24.70 \\
QAGC-LSTM $_{\mathrm{R}}$ & 23.368 & 87.18 & 39.43 & $\mathbf{7 9 . 1 3}$ & 24.70 \\
\hline DGNN & 4.0789 & 89.68 & $\mathbf{8 9 . 9 2}$ & 72.35 & $\mathbf{7 1 . 3 0}$ \\
QDGNN $_{\text {QDGNN }}$ & 4.075 & 86.55 & 41.06 & $\mathbf{8 2 . 2 6}$ & 27.13 \\
\hline \hline
\end{tabular}

QMLP has 10 layers, in which the middle layer maps from $\mathbb{H}^{256}$ to $\mathbb{H}^{256}$, and its input and output are 64 quaternions, respectively.

We implement our fast QPU based on the acceleration strategy 3 proposed in Section 4.2, in which strategy 2 will be used as its feed-forward part. The implementation is based on CUDA. To demonstrate the efficiency of our fast QPU, we compare it with the naive QPU implementation using the AutoGrad algorithm provided by PyTorch [57]. Given a set of quaternions as inputs, we test the runtime and the memory cost of the two implementations and show the average of their performance over 10 trials in Table 1. All experiments are conducted in a stable server environment. The GPU used in this experiment is GeForce RTX $2080 \mathrm{Ti}$, and the CPU model is Intel(R) Xeon(R) E5-2678 v3 2.50GHz. Compared to the naive QPU based on AutoGrad, our fast QPU is nearly $10 \times$ faster in the feed-forward step. Moreover, the acceleration ratio is strengthened with the increase of the network depth, which achieves $20 \times$ acceleration for the 10-layer QMLP. At the same time, our fast QPU also reduces the backward time and memory consumption to $75 \%$ on average, which has a significant effect.

\subsection{Experiments on real-world skeleton modeling}

To demonstrate the effectiveness of our QPU-based models in skeleton modeling tasks, we consider two real-world skeleton datasets: the NTU dataset [16] for human action recognition and the FPHA dataset [58] for hand action recognition. The NTU dataset provides 56,578 human skeleton sequences performed by 40 actors belonging to 60 classes. The cross-view protocol is used in our experiments. We use 37,646 sequences for training and the remaining 18,932 sequences for testing. The FPHA dataset provides hand skeletons recorded with magnetic sensors and computed by inverse kinematics, which consists of 1,175 action videos belonging to 45 categories and performed by 6 actors. Following the setting in [58], we use 600 sequences for training and 575 sequences for testing.

For these two datasets, we implement three real-valued models as our baselines and propose their quaternion versions by replacing their key modules with QPUs.
- RMLP-LSTM v.s. QMLP-LSTM: The RMLP-LSTM is composed of a two-layer MLP and a one-layer LSTM. The MLP merges each frame into a feature vector and is shared between frames. Features of each frame are then fed into the LSTM layer. We average the outputs of the LSTM at different steps and feed it into a classifier. Replacing the two FC layers of the RMLP-LSTM with two QPU-based layers leads to our QMLP-LSTM.

- AGC-LSTM v.s. QAGC-LSTM: The AGC-LSTM in [20] first uses an FC layer on each joint to increase the feature dimension. The features are fed into a three-layer graph convolutional network. Our QAGC-LSTM replaces the input FC layer of the AGC-LSTM with a QPU-based layer, whose input includes two channels corresponding to the quaternions of each joint node and its parent node.

- DGNN v.s. QDGNN: The DGNN in [19] applies ten directed graph network (DGN) blocks to merge both node features and edge features and extracts temporal information via a temporal convolution across the DGCNs. Our QDGNN replaces the first DGN of the DGNN with the proposed QMP layer shown in Fig. 5b and (25). For the input of our QDGNN, the node features are the unit quaternions corresponding to the joint rotations, i.e., $\left\{q_{i}\right\}_{i=1}^{N}$, and the edge features are the differences between joint rotations, i.e., $q_{i} \otimes q_{j}^{*}$ for the edge $i \rightarrow j$.

For each of the above QNNs, we consider its variant that focuses on the rotation-invariance, including QMLP-LSTM Q $_{\mathbf{R}}$, QAGC$\mathbf{L S T M}_{\mathbf{R}}$, and $\mathbf{Q D G N N} \mathbf{R}_{\mathbf{R}}$. For the QNNs (and their variants), the numbers of model parameters are almost the same as those of the corresponding real-valued models. The experimental results are shown in Table 2. We can find that the performance of our QPU-based models is at least comparable to the baselines in the NR scenario. In the more challenging AR scenario, ${ }^{3}$ the variants of the QNNs that purely output rotation-invariant features retain high accuracy consistently, while the performance of the baselines degrades a lot. These phenomena are consistent to what we observed in the synthetic experiments, which further verify the usefulness of our models.

\subsection{Experiments on real-world point cloud modeling}

We further test our QPU-based models for rotation-invariant point cloud classification on two datasets: the ModelNet40 and the ModelNet10 proposed in [59]. These two datasets contain normalized point clouds sampled from 40 and 10 types of CAD models, respectively. To maintain the consistency of the input, 1024 points will be randomly sampled from the point set for each inference.

3. The random rotations are sampled uniformly from the rotation space. For each rotation, its three parameters $\left\{\frac{\theta}{2}, \alpha, \beta\right\} \sim \boldsymbol{U}(0, \pi)$, where $\theta$ represents the rotation angle, $\alpha / \beta$ represent the angle between the rotation axis and the $\mathrm{z} / \mathrm{x}$-axis. 
TABLE 3: Comparisons on testing accuracy (\%) on ModelNet40 and ModelNet10.

\begin{tabular}{l|cc|cc}
\hline \hline \multirow{2}{*}{ Model } & \multicolumn{2}{|c|}{ ModelNet40 } & \multicolumn{2}{c}{ ModelNet10 } \\
& NR & AR & NR & AR \\
\hline PointNet++ & 88.4 & 22.0 & 79.1 & 33.8 \\
QPointNet++ & 89.4 & 21.7 & 80.9 & 31.8 \\
QPointNet++ & $\mathbf{9 0 . 2}$ & 21.2 & $\mathbf{8 3 . 8}$ & 31.9 \\
QPointNet++R & 81.6 & 81.8 & 66.9 & 67.1 \\
QPointNet++ & 84.0 & $\mathbf{8 4 . 0}$ & 67.5 & $\mathbf{6 8 . 5}$ \\
\hline RSCNN & 92.38 & 21.23 & $\mathbf{9 1 . 8 5}$ & 32.82 \\
QRSCNN & 92.42 & 20.70 & 90.97 & 37.11 \\
QRSCNN $_{I}$ & $\mathbf{9 2 . 5 0}$ & 19.45 & 89.54 & 34.58 \\
QRSCNN $_{R}$ & 86.59 & 86.14 & 78.85 & 77.97 \\
QRSCNN $_{\text {RI }}$ & 86.83 & $\mathbf{8 6 . 8 7}$ & 79.24 & $\mathbf{7 9 . 1 8}$ \\
\hline \hline
\end{tabular}

TABLE 4: Comparison with other rotation-invariant networks on testing accuracy $(\%)$.

\begin{tabular}{l|cc}
\hline \hline \multirow{2}{*}{ Model } & \multicolumn{2}{|c}{ ModelNet40 } \\
& NR & AR \\
\hline QE-Net & 74.43 & 74.07 \\
REQNN (PointNet++) & 63.95 & 63.95 \\
REQNN (DGCNN) & 83.03 & 83.03 \\
\hline QPointNet++RI $_{\text {QRSCNN }_{\text {RI }}}^{84.0}$ & 84.0 \\
PR & $\mathbf{8 6 . 8 7}$ & $\mathbf{8 6 . 8 7}$ \\
\hline
\end{tabular}

In this experiment, we consider two state-of-the-art real-valued point cloud representation models and propose their quaternion version with the help of our QPUs. Specifically, we have

- PointNet++ v.s. QPointNet++: PointNet++ [23] is a typical point-wise point cloud classification network, ${ }^{4}$ which is composed of three set-abstraction layers for feature extraction and one FC layer for classification. Our QPointNet++ replaces the first set abstraction layer with the QSA layer we defined before.

- RSCNN v.s. QRSCNN: RSCNN [26] is a lightweight implementation of a convolution-based point cloud classification model, whose overall structure is similar to PointNet++. The main difference is that the MLP used in the set abstraction layer of PointNet++ is replaced with the RSConv layer. Our QRSCNN replaces the RSConv with the QRSConv defined in (26).

As we mentioned in Section 5.1, the above QNNs need to sort the quaternions associated with the neighborhood of the centroid point. In this experiment, we apply the rotation-based sorting method to design their QSA layer. The superiority of the rotationbased sorting to the other two sorting methods (i.e., the anglebased and the distance-based methods) will be verified in the next subsection. Additionally, for each of the above QNNs, besides the variant studied in the experiments of skeleton modeling, we further consider two more variants: QPointNet++ ${ }_{I}$ and $\mathbf{Q R S C N N}_{\mathbf{I}}$ merely output rotation-equivariant features, while $\mathbf{Q P o i n t N e t + + _ { \mathbf { R I } }}$ and $\mathbf{Q R S C N N} \mathbf{N I}_{\mathbf{R}}$ output rotation-invariant features that leverage real and imaginary parts jointly.

Experiment results of our models and their competitors are shown in Table 3. We can find that our QNNs outperform the real-value models in most situations, which further verifies the effectiveness of our QPU. Additionally, in the NR scenario, the models purely considering the rotation-equivariant features (i.e., QPointNet++ ${ }_{I}$ and $\mathrm{QRSCNN}_{\mathrm{I}}$ ) achieve the best performance. In the AR scenario, however, the models emphasizing their rotation-

4. https://github.com/erikwijmans/Pointnet2_PyTorch.
TABLE 5: The impact of angle-axis map on accuracy (\%) with FPHA as dataset.

\begin{tabular}{c|c|c}
\hline \hline Model & w. angle-axis map & w/o. angle-axis map \\
\hline QMLP-LSTM & $\mathbf{7 6 . 1 7}$ & 73.04 \\
QAGC-LSTM & $\mathbf{7 9 . 1 3}$ & 75.65 \\
QDGNN & $\mathbf{7 6 . 3 5}$ & 75.30 \\
\hline
\end{tabular}

TABLE 6: The impact of sorting methods on testing accuracy(\%)

\begin{tabular}{l|c|cc}
\hline \hline \multirow{2}{*}{ Model } & \multirow{2}{*}{ Sorting method } & \multicolumn{2}{|c}{ ModelNet40 } \\
& & NR & AR \\
\hline \multirow{3}{*}{ QPointNet++ } & Rotation-based & $\mathbf{8 1 . 6 0}$ & $\mathbf{8 1 . 8 0}$ \\
& Angle-based & 80.16 & 80.32 \\
& Distance-based & 80.28 & 79.84 \\
\hline \hline
\end{tabular}

invariance (i.e., QPointNet++ ${ }_{\mathrm{RI}}$ and $\mathrm{QRSCNN}_{\mathrm{RI}}$ ) achieve the best performance in the slight sacrifice of their accuracy in the NR scenario. Specifically, for a QPU-based QNN, its rotationequivariant outputs (the imaginary parts of the output quaternions) are informative for representing 3D data. Therefore, purely considering the rotation-invariant outputs (the real parts of the output quaternions) may lead to obvious degradation in performance (see the results of QPointNet++ $t_{R}$ and $\mathrm{QRSCNN}_{\mathrm{R}}$ ). However, these rotation-equivariant outputs are sensitive to the noisy rotations, whose performance in the AR scenario is questionable. As a result, QPointNet++ ${ }_{\mathrm{RI}}$ and $\mathrm{QRSCNN}_{\mathrm{RI}}$ consider both real and imaginary parts of the outputs, which achieve a trade-off between the representation power and the rotation robustness. This interesting phenomenon demonstrates the disentanglement of rotation-invariance and rotation-equivariance achieved by our model. Moreover, it implies that we should apply different models according to the noise-level of data and the requirements of application scenarios.

To further demonstrate the superiority of our QPU-based models, we compare our QPointNet++ ${ }_{\mathrm{RI}}$ and $\mathrm{QRSCNN}_{\mathrm{RI}}$ with other quaternion-based neural networks, including the QE-Net in [31] and the REQNN in [30]. These two baselines also output rotation-invariant features for 3D data modeling, and the REQNN can be developed based on the PointNet++ and the DGCNN, respectively. We test the models on the ModelNet40 dataset, and Table 4 shows their comparisons. ${ }^{5}$ We can find that our QPUbased models consistently outperform the baselines in both testing scenarios.

\subsection{Ablation study}

Usefulness of the angle-axis map. In the above experiments, our QNNs use the angle-axis map (or its variants) to convert quaternion outputs to real ones. Without this map, we have to treat each output quaternion as a 4-dimensional real-valued vector directly. To quantitatively demonstrate the usefulness of the angleaxis map, we implement experiments on the FPHA dataset to compare the performance of QMLP-LSTM and QAGC-LSTM with/without the map, as shown in Table 5. The results verify our claim in Section 5.2: for our QNNs, their performance boosts a lot when we connect their QPU-based layers with the following real-valued layers through the angle-axis map.

Rationality of rotation-based sorting. When modeling point clouds, our QNNs apply the rotation-based sorting method to permute the neighbor points of each centroid. To demonstrate

5. Since QE-Net and REQNN did not claim their performances on ModelNet10, we only chose ModelNet40 for comparison. 
the rationality of this setting, we apply different sorting methods to QPointNet $++_{\mathrm{R}}$, and test their performance on the ModelNet40 dataset. The experimental results in Table. 6 show that the rotationbased sorting method can obtain the best results in both NR and AR testing scenarios.

\section{Conclusion}

In this paper, we have proposed a novel and efficient quaternionbased neuron model called fast QPU, which can be used for various $3 \mathrm{D}$ tasks to disentangle $3 \mathrm{D}$ representations into rotationinvariant and rotation-equivariant parts, respectively. Experiments show that applying the QPU-based neural networks can obtain strong rotation robustness at the limited expense of accuracy, which outperforms many real-valued models in challenging situations. Compared with other quaternion-based work, our QPUbased models demonstrate their superiority as well. When implementing the fast QPU, we developed a new tree-structured indexing process and leverage the properties of the Hamilton product to accelerate the forward and backward processes at the same time. In the future, we will consider more applications suitable for our QPU-based models. In the aspect of modeling, we will make attempts to extend our model from the rotation group $\mathbb{S O}(3)$ to more complicated groups, e.g., $\mathbb{S E}(3)$.

\section{REFERENCES}

[1] C. R. Qi, W. Liu, C. Wu, H. Su, and L. J. Guibas, "Frustum pointnets for $3 \mathrm{~d}$ object detection from rgb-d data," in Proceedings of the IEEE conference on computer vision and pattern recognition, 2018, pp. 918 927.

[2] Y. Zhou and O. Tuzel, "Voxelnet: End-to-end learning for point cloud based 3d object detection," in Proceedings of the IEEE conference on computer vision and pattern recognition, 2018, pp. 4490-4499.

[3] T. Whelan, S. Leutenegger, R. Salas-Moreno, B. Glocker, and A. Davison, "Elasticfusion: Dense slam without a pose graph." Robotics: Science and Systems, 2015.

[4] V. Bloom, D. Makris, and V. Argyriou, "G3d: A gaming action dataset and real time action recognition evaluation framework," in 2012 IEEE Computer Society Conference on Computer Vision and Pattern Recognition Workshops. IEEE, 2012, pp. 7-12.

[5] D. Pavllo, D. Grangier, and M. Auli, "Quaternet: A quaternion-based recurrent model for human motion," in British Machine Vision Conference, 2018.

[6] R. Vemulapalli, F. Arrate, and R. Chellappa, "Human action recognition by representing $3 \mathrm{~d}$ skeletons as points in a lie group," in Proceedings of the IEEE conference on computer vision and pattern recognition, 2014, pp. 588-595.

[7] P. Zhang, C. Lan, J. Xing, W. Zeng, J. Xue, and N. Zheng, "View adaptive neural networks for high performance skeleton-based human action recognition," IEEE transactions on pattern analysis and machine intelligence, vol. 41, no. 8, pp. 1963-1978, 2019.

[8] T. S. Cohen, M. Geiger, J. Köhler, and M. Welling, "Spherical cnns," in International Conference on Learning Representations, 2018.

[9] C. Esteves, C. Allen-Blanchette, A. Makadia, and K. Daniilidis, "Learning so (3) equivariant representations with spherical cnns," in Proceedings of the European Conference on Computer Vision (ECCV), 2018, pp. 52-68.

[10] M. Weiler, M. Geiger, M. Welling, W. Boomsma, and T. Cohen, "3d steerable cnns: learning rotationally equivariant features in volumetric data," in Proceedings of the 32nd International Conference on Neural Information Processing Systems, 2018, pp. 10402-10 413.

[11] C. Chen, G. Li, R. Xu, T. Chen, M. Wang, and L. Lin, "Clusternet: Deep hierarchical cluster network with rigorously rotation-invariant representation for point cloud analysis," in Proceedings of the IEEE/CVF Conference on Computer Vision and Pattern Recognition, 2019, pp. 4994-5002.

[12] N. Thomas, T. Smidt, S. Kearnes, L. Yang, L. Li, K. Kohlhoff, and P. Riley, "Tensor field networks: Rotation-and translation-equivariant neural networks for 3d point clouds," arXiv preprint arXiv:1802.08219, 2018.
[13] C. R. Qi, H. Su, K. Mo, and L. J. Guibas, "Pointnet: Deep learning on point sets for 3d classification and segmentation," in Proceedings of the IEEE conference on computer vision and pattern recognition, 2017, pp. $652-660$.

[14] Z. Huang, C. Wan, T. Probst, and L. Van Gool, "Deep learning on lie groups for skeleton-based action recognition," in Proceedings of the IEEE conference on computer vision and pattern recognition, 2017, pp. 60996108.

[15] X. S. Nguyen, L. Brun, O. Lézoray, and S. Bougleux, "A neural network based on spd manifold learning for skeleton-based hand gesture recognition," in Proceedings of the IEEE/CVF Conference on Computer Vision and Pattern Recognition, 2019, pp. 12036-12045.

[16] A. Shahroudy, J. Liu, T.-T. Ng, and G. Wang, "Ntu rgb+ d: A large scale dataset for $3 \mathrm{~d}$ human activity analysis," in Proceedings of the IEEE conference on computer vision and pattern recognition, 2016, pp. 10101019.

[17] J. Liu, A. Shahroudy, D. Xu, and G. Wang, "Spatio-temporal 1stm with trust gates for 3d human action recognition," in European conference on computer vision. Springer, 2016, pp. 816-833.

[18] S. Yan, Y. Xiong, and D. Lin, "Spatial temporal graph convolutional networks for skeleton-based action recognition," in Thirty-second AAAI conference on artificial intelligence, 2018.

[19] L. Shi, Y. Zhang, J. Cheng, and H. Lu, "Skeleton-based action recognition with directed graph neural networks," in Proceedings of the IEEE/CVF Conference on Computer Vision and Pattern Recognition, 2019, pp. 7912-7921.

[20] C. Si, W. Chen, W. Wang, L. Wang, and T. Tan, "An attention enhanced graph convolutional lstm network for skeleton-based action recognition," in Proceedings of the IEEE/CVF Conference on Computer Vision and Pattern Recognition, 2019, pp. 1227-1236.

[21] M. Korban and X. Li, "Ddgen: A dynamic directed graph convolutional network for action recognition," in European Conference on Computer Vision. Springer, 2020, pp. 761-776.

[22] Y. Chen, Z. Zhang, C. Yuan, B. Li, Y. Deng, and W. Hu, "Channelwise topology refinement graph convolution for skeleton-based action recognition," in Proceedings of the IEEE/CVF International Conference on Computer Vision, 2021, pp. 13359-13 368.

[23] C. R. Qi, L. Yi, H. Su, and L. J. Guibas, "Pointnet++ deep hierarchical feature learning on point sets in a metric space," in Proceedings of the 31st International Conference on Neural Information Processing Systems, 2017, pp. 5105-5114.

[24] H. Zhao, L. Jiang, C.-W. Fu, and J. Jia, "Pointweb: Enhancing local neighborhood features for point cloud processing," in Proceedings of the IEEE/CVF Conference on Computer Vision and Pattern Recognition, 2019, pp. 5565-5573.

[25] W. Wu, Z. Qi, and L. Fuxin, "Pointconv: Deep convolutional networks on $3 \mathrm{~d}$ point clouds," in Proceedings of the IEEE/CVF Conference on Computer Vision and Pattern Recognition, 2019, pp. 9621-9630.

[26] Y. Liu, B. Fan, S. Xiang, and C. Pan, "Relation-shape convolutional neural network for point cloud analysis," in Proceedings of the IEEE/CVF Conference on Computer Vision and Pattern Recognition, 2019, pp. 8895-8904.

[27] M. Xu, R. Ding, H. Zhao, and X. Qi, "Paconv: Position adaptive convolution with dynamic kernel assembling on point clouds," in Proceedings of the IEEE/CVF Conference on Computer Vision and Pattern Recognition, 2021, pp. 3173-3182.

[28] M. Jaderberg, K. Simonyan, A. Zisserman et al., "Spatial transformer networks," Advances in neural information processing systems, vol. 28, pp. 2017-2025, 2015.

[29] R. Vemulapalli and R. Chellapa, "Rolling rotations for recognizing human actions from 3d skeletal data," in Proceedings of the IEEE conference on computer vision and pattern recognition, 2016, pp. 44714479.

[30] W. Shen, B. Zhang, S. Huang, Z. Wei, and Q. Zhang, "3d-rotationequivariant quaternion neural networks," in Computer Vision-ECCV 2020: 16th European Conference, Glasgow, UK, August 23-28, 2020, Proceedings, Part XX 16. Springer, 2020, pp. 531-547.

[31] Y. Zhao, T. Birdal, J. E. Lenssen, E. Menegatti, L. Guibas, and F. Tombari, "Quaternion equivariant capsule networks for $3 \mathrm{~d}$ point clouds," in European Conference on Computer Vision. S Springer, 2020, pp. 1-19.

[32] E. Bayro-Corrochano, "The theory and use of the quaternion wavelet transform," Journal of Mathematical Imaging and Vision, vol. 24, no. 1, pp. 19-35, 2006.

[33] J. Zhou, Y. Xu, and X. Yang, "Quaternion wavelet phase based stereo matching for uncalibrated images," Pattern Recognition Letters, vol. 28 , no. 12, pp. 1509-1522, 2007. 
[34] X. Ma, Y. Xu, L. Song, X. Yang, and H. Burkhardt, "Color image watermarking using local quaternion fourier spectral analysis," in 2008 IEEE International Conference on Multimedia and Expo. IEEE, 2008, pp. 233-236.

[35] L. Yu, Y. Xu, H. Xu, and H. Zhang, "Quaternion-based sparse representation of color image," in 2013 IEEE International Conference on Multimedia and Expo (ICME). IEEE, 2013, pp. 1-7.

[36] Y. Xu, L. Yu, H. Xu, H. Zhang, and T. Nguyen, "Vector sparse representation of color image using quaternion matrix analysis," IEEE Transactions on image processing, vol. 24, no. 4, pp. 1315-1329, 2015.

[37] A. Szczesna, A. Świtoński, J. Słupik, H. Zghidi, H. Josiński, and K. Wojciechowski, "Quaternion lifting scheme applied to the classification of motion data," Information Sciences, 2018.

[38] K. Shoemake, "Animating rotation with quaternion curves," in Proceedings of the 12th annual conference on Computer graphics and interactive techniques, 1985, pp. 245-254.

[39] T. Isokawa, T. Kusakabe, N. Matsui, and F. Peper, "Quaternion neural network and its application," in International conference on knowledgebased and intelligent information and engineering systems. Springer, 2003, pp. 318-324.

[40] C. J. Gaudet and A. S. Maida, "Deep quaternion networks," in 2018 International Joint Conference on Neural Networks (IJCNN). IEEE, 2018, pp. 1-8.

[41] T. Parcollet, M. Morchid, and G. Linarès, "Quaternion convolutional neural networks for heterogeneous image processing," in ICASSP 2019. 2019 IEEE International Conference on Acoustics, Speech and Signal Processing (ICASSP). IEEE, 2019, pp. 8514-8518.

[42] X. Zhu, Y. Xu, H. Xu, and C. Chen, "Quaternion convolutional neural networks," in Proceedings of the European Conference on Computer Vision (ECCV), 2018, pp. 631-647.

[43] T. Parcollet, M. Ravanelli, M. Morchid, G. Linarès, C. Trabelsi, R. De Mori, and Y. Bengio, "Quaternion recurrent neural networks," in International Conference on Learning Representations, 2018.

[44] Y. Tay, A. Zhang, A. T. Luu, J. Rao, S. Zhang, S. Wang, J. Fu, and S. C. Hui, "Lightweight and efficient neural natural language processing with quaternion networks," in Proceedings of the 57th Annual Meeting of the Association for Computational Linguistics, 2019, pp. 1494-1503.

[45] Y. Liu, Y. Zheng, J. Lu, J. Cao, and L. Rutkowski, "Constrained quaternion-variable convex optimization: a quaternion-valued recurrent neural network approach," IEEE transactions on neural networks and learning systems, vol. 31, no. 3, pp. 1022-1035, 2019.

[46] Z. Guo, J. Zhao, L. Jiao, X. Liu, and F. Liu, "A universal quaternion hypergraph network for multimodal video question answering," IEEE Transactions on Multimedia, 2021.

[47] Z. Li, Q. Xu, Y. Jiang, X. Cao, and Q. Huang, "Quaternion-based knowledge graph network for recommendation," in Proceedings of the 28th ACM International Conference on Multimedia, 2020, pp. 880-888.

[48] X. Zhang, S. Qin, Y. Xu, and H. Xu, "Quaternion product units for deep learning on 3d rotation groups," in Proceedings of the IEEE/CVF Conference on Computer Vision and Pattern Recognition, 2020, pp. 7304-7313.

[49] E. B. Dam, M. Koch, and M. Lillholm, Quaternions, interpolation and animation. Citeseer, 1998, vol. 2.

[50] J. Voight, Quaternion algebras. Springer Nature, 2021.

[51] F. Rosenblatt, "The perceptron: a probabilistic model for information storage and organization in the brain." Psychological review, vol. 65, no. 6, p. 386, 1958.

[52] A. Paszke, S. Gross, S. Chintala, G. Chanan, E. Yang, Z. DeVito, Z. Lin, A. Desmaison, L. Antiga, and A. Lerer, "Automatic differentiation in pytorch," in Proceedings of the Neural Information Processing Systems, 2017.

[53] E. E. Santos, "Optimal and efficient algorithms for summing and prefix summing on parallel machines," Journal of Parallel and Distributed Computing, vol. 62, no. 4, pp. 517-543, 2002.

[54] X. Glorot and Y. Bengio, "Understanding the difficulty of training deep feedforward neural networks," in Proceedings of the thirteenth international conference on artificial intelligence and statistics. JMLR Workshop and Conference Proceedings, 2010, pp. 249-256.

[55] D. Q. Huynh, "Metrics for 3d rotations: Comparison and analysis," Journal of Mathematical Imaging and Vision, vol. 35, no. 2, pp. 155164, 2009.

[56] D. P. Kingma and J. Ba, "Adam: A method for stochastic optimization," in In Proceedings of the 3rd International Conference on Learning Representations, 2015.

[57] A. Paszke, S. Gross, F. Massa, A. Lerer, J. Bradbury, G. Chanan, T. Killeen, Z. Lin, N. Gimelshein, L. Antiga et al., "Pytorch: An imperative style, high-performance deep learning library," Advances in neural information processing systems, vol. 32, pp. 8026-8037, 2019.

[58] G. Garcia-Hernando, S. Yuan, S. Baek, and T.-K. Kim, "First-person hand action benchmark with rgb-d videos and $3 \mathrm{~d}$ hand pose annotations," in Proceedings of the IEEE conference on computer vision and pattern recognition, 2018, pp. 409-419.

[59] Z. Wu, S. Song, A. Khosla, F. Yu, L. Zhang, X. Tang, and J. Xiao, "3d shapenets: A deep representation for volumetric shapes," in Proceedings of the IEEE conference on computer vision and pattern recognition, 2015, pp. 1912-1920.

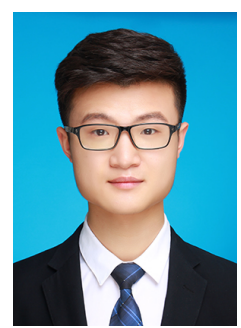

Shaofei Qin is currently completing the M.S. degree in Information Engineering from Shanghai Jiao Tong University, China and is expected to graduate in 2023. His recent research work mainly involves the computational optimization of neural networks and quaternion neural networks.

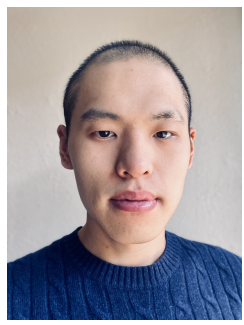

Xuan Zhang received the B.S. degree in information engineering and the M.E. degree in electronics and communication engineering from Shanghai Jiao Tong University, Shanghai, China, in 2017 and 2020, respectively. He is currently working toward the Ph.D. degree in the Department of Computer Science and Engineering, Texas A\&M University, College Station, Texas. His research interests include machine learning, deep learning, and data mining.

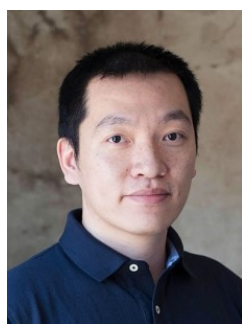

Hongteng $\mathbf{X u}$ is an Associate Professor (Tenure-Track) in the Gaoling School of Artificial Intelligence, Renmin University of China. From 2018 to 2020, he was a senior research scientist in Infinia ML Inc. In the same time period, he is a visiting faculty member in the Department of Electrical and Computer Engineering, Duke University. He received his Ph.D. from the School of Electrical and Computer Engineering at Georgia Institute of Technology (Georgia Tech) in 2017. His research interests include machine learning and its applications, especially optimal transport theory, sequential data modeling and analysis, deep learning techniques, and their applications in computer vision and data mining.

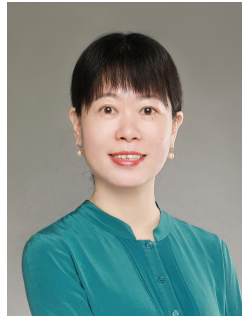

Yi Xu received the B.S. and M.S. degrees from the Nanjing University of Science and Technology, Nanjing, China, in 1996 and 1999, respectively, and the Ph.D. degree from Shanghai Jiao Tong University, Shanghai, China, in 2005. She is currently an Associate Professor with the Department of Electronic Engineering, Institute of Image Communication and Network Engineering, Shanghai Jiao Tong University. Her research interests include image processing, video analysis, and quaternion-based neural network. 